\title{
LINE SEGMENTS WHICH ARE UNIONS OF TESSELLATION EDGES
}

\author{
RICHARD COWAN ${ }^{\otimes}, 1$ AND VIOLA WEISS ${ }^{2}$ \\ ${ }^{1}$ School of Mathematics and Statistics, University of Sydney, NSW 2006, Australia.; ${ }^{2}$ Ernst-Abbe-Hochschule, \\ Jena, Germany. \\ e-mail: richard.cowan@sydney.edu.au,viola.weiss@eah-jena.de \\ (Received September 8, 2016; revised January 19, 2018; accepted January 29, 2018)
}

\begin{abstract}
Planar tessellation structures occur in material science, geology (in rock formations), physics (of foams, for example), biology (especially in epithelial studies) and in other sciences. Their mathematical and statistical study has many aspects to consider. In this paper, line-segments which are either a tessellation edge or a finite union of edges are studied. Our focus is on a sub-class of such line-segments - those we call M-segments - that are not contained in a longer union of edges. These encompass the so-called I-segments that have arisen in many recent tessellation models. We study the expected numbers of edges and cell-sides contained in these M-segments, and the prevalence of these entities. Many examples and figures, including some based on tessellation nesting and superposition, illustrate the theory. M-segments are much more prevalent when a tessellation is not side-to-side, so our paper has theoretical connections with the recent IAS paper by Cowan and Thäle (2014); that paper characterised non side-to-side tessellations.
\end{abstract}

Keywords: combinatorial topology, edge types, planar tessellations, STIT tessellation, stochastic geometry, superposition/nesting.

\section{INTRODUCTION}

In this paper we study geometric structures seen in random planar tessellations: namely line-segments which are either a tessellation edge or a finite union of edges. Collectively, we call these structures tessellation segments (see Definition 1). Of course, to achieve a finite union which is a line-segment, the edges contributing to the union must be collinear.

Some of these tessellation segments are maximal in the sense of Definition 1.

Definition 1. A closed and bounded line-segment which is either an edge of the planar tessellation or a finite union of the tessellation's edges is called a tessellation segment. An M-segment is a maximal tessellation segment. It is 'maximal' in the sense that it is not contained in a longer tessellation segment.

It is the class of M-segments that attracts our main attention in this paper, though we also look at a subclass, the I-segments - which have been discussed in earlier studies.

Definition 2. A vertex of the tessellation is called a $\pi$-vertex if and only if the edges emanating from the vertex subtend one angle of $\pi$. An I-segment is an $\mathrm{M}$ segment with $\pi$-vertices at both ends. So $\mathrm{I} \subseteq \mathrm{M}$.

An M-segment may have non- $\pi$ vertices (written $\bar{\pi}$-vertices) at its two end points (termini); only if both of these termini are $\pi$-vertices does the line-segment (together with a small neighbourhood of its termini) look like the letter I, hence the name I-segment. Fig. 1 shows examples of $\pi$-vertices, I-segments and $M$ segments.

The role of $\pi$-vertices was first discussed in Cowan (1978; 1980). The name I-segment was first used in papers by Mackisack and Miles (1996) and Miles and Mackisack (2002) dealing with particular models for planar tessellations.

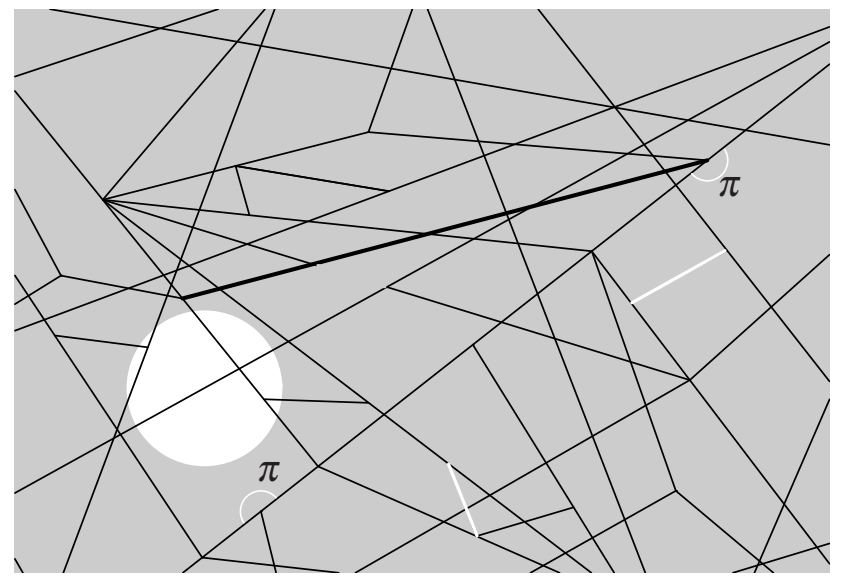

Fig. 1. The bold black line-segment is an M-segment that is not an I-segment. The two white segments, one comprising two edges and the other only one, are Isegments. Two of the many $\pi$-vertices in the figure have their subtended $\pi$ angle marked. The white disk is discussed in Remark 2. 
I-segments were also analysed in the context of the so-called STIT model by Mecke et al. (2007; 2011) and Cowan (2013). In this model and those of Miles and Mackisack, all vertices are $\pi$-vertices. Some other models, for example the Gilbert model (Gilbert, 1967) and various iteratively-divided models in Cowan (2010) also have this feature. So there were no Msegments that were not I-segments in these earlier studies. In other words, $M \backslash I=\emptyset$ for the models whose I-segments have to date been discussed.

Our current study is model-free; we only impose stationarity of the planar tessellation (an invariance of the tessellation's statistical properties under translation) and convexity of its cells - so they are convex polygons - together with a local-finiteness condition (any bounded domain intersects a finite number of cells). Furthermore we assume that both the vertex intensity (the mean number of vertices per unit area) and the mean length of the typical edge are positive and finite. In this general context, which embraces all stationary planar tessellations except those where no $\mathrm{M}$-segments exist, we derive formulae for the composition of the typical M-segment: the expected number of edges and the expected number of cell-sides lying in the M-segment. Also we find these results for superposition and nesting of tessellations. With no further assumptions about the tessellation structure, one cannot obtain distributional results, but these mean-value results are of interest and new.

\section{BASIC TERMINOLOGY AND NOTATION}

Our discussion adopts the language and concepts of the earlier papers, Weiss and Cowan (2011) and Cowan and Thäle (2014). A tessellation of the plane is a collection of compact convex polygonal cells which cover the plane and overlap only on cell boundaries. The union of the cell boundaries is called the tessellation frame. Each cell has sides and corners, these being respectively the 1 -faces and 0 -faces of the polygon; they lie on the frame. The union (taken over all cells) of cell corners is a collection of points in the plane called the vertices of the tessellation. Those line segments which are contained in the frame, have a vertex at each end and no vertices in their interior ${ }^{1}$ are called edges of the tessellation.

Remark 1. It is useful here, now that the concept of a cell side has been introduced, to note that a $\pi$-vertex is a vertex which lies in the interior of a cell side. Clearly, $\bar{\pi}$-vertices do not.

As discussed in Weiss and Cowan (2011), the focus of attention in many studies of planar tessellations and tilings has been the side-to-side case.

Definition 3. A tessellation is side-to-side if, for every cell, each side of that cell coincides with the side of another cell. Equivalently, one can say that a tessellation is side-to-side if and only if it has no $\pi$ vertices. $^{2}$

In the non side-to-side case, the distinction between edges and sides is important. We note that some writers use the term edge-to-edge where we say side-to-side; we see no logic in the edge-to-edge terminology once the distinction between edges and sides is recognised.

Primitive and non-primitive elements: The tessellation's primitive geometric elements, treated as compact domains, are the vertices, edges and cells. The collections of these elements are denoted by $\mathrm{V}$, $\mathrm{E}$ and $\mathrm{Z}$ (for Zellen) respectively (and we note that $\mathrm{V}, \mathrm{E}$ and $\mathrm{Z}$ are sets, because no element appears more than once). Other compact geometric elements will be introduced and the collection of these will also be denoted by a non-sloping upper-case letter - for example, $\mathrm{S}$ and $\mathrm{C}$ for all sides and corners of cells, respectively. As seen above, M and I are the sets of $\mathrm{M}$-segments and I-segments respectively. Tessellation frames may contain infinite-length full lines and the set of these full ${ }^{3}$ lines is called L.

We note that, for those elements which are not primitive but are instead derived from a primitive element or from some other feature of the tessellation, then the collection might have elements which appear more than once. We call these collections multisets. For example, there will be many corners located at each vertex; thus we speak of the multiset $\mathrm{C}$ of corners.

Likewise, we have a multiset $\mathrm{S}$ of sides. In sideto-side tessellations, every element in $S$ equals another member of $\mathrm{S}$, as befits the terminology side-to-side. This may be true for some (but not all) members of $S$ in the non side-to-side case. In general, cell sides are

\footnotetext{
${ }^{1}$ Here the interior of a line-segment, or indeed of any object $x$ of lower dimension than the space of the tessellation, is defined using the relative topology on $x$. So when we use the word 'interior' in such contexts, we mean 'relative interior'.

2 A side-to-side tessellation is obviously corner-to-corner in the sense that, for every cell, each corner of that cell is the corner of another cell. These concepts are encompassed in the $d$-dimensional discussion of Schneider and Weil (2008), page 447, where the general phrase face-to-face is used - see also Lemma 1.1 of Cowan and Weiss (2015).

${ }^{3}$ Full lines extend infinitely in both directions. Later we discuss half-lines, which extend infinitely in only one direction - showing (via Lemma 3) that these don't exist in stationary tessellations. At this stage, we merely say that $L$ does not includes half-lines and, from now, the word 'line' means 'full line'.
} 
line-segments which equal an edge or a finite union of edges. So S, like M and I, only contains elements which are tessellation segments.

Generic (multi)sets of geometric objects are denoted by $X$ and $Y$. Sub(multi)sets of $X$ are denoted by $X[\cdot]$, with the contents of the $[\cdot]$ being a suitably suggestive symbol introduced in an ad hoc manner. For example, we denote the subset of $\pi$-vertices by $\mathrm{V}[\pi]$, the subset of non $\pi$-vertices ( $\bar{\pi}$-vertices) by $\mathrm{V}[\bar{\pi}]$, the 'sub-multiset' of corners which lie on $\pi$-vertices by $C[\pi]$ and the subset of vertices which lie on exactly $j$ lines $\in \mathrm{L}$ by $\mathrm{V}[\mathrm{L}, j]$.

Remark 2. From any multiset, a reduced set of elements ignoring all multiplicities can be constructed - also a function mapping this reduced set into the positive integers can indicate the multiplicities. Thus a multiset might be called a marked set, the 'mark' indicating the multiplicity of an element. With this device, calculations can proceed quite logically, though it would be notationally complicated if spelt out completely with algebraic symbols for reduced sets and mark functions. We avoid such detail as our calculations with multisets are intuitively clear. For example, if we asked for the number of sides intersecting the white disk in Fig. 1, we expect our reader to answer: 16. For corners: 8, counting the multiplicities in $\mathrm{C}$ at three vertices.

In the early part of the twentieth century, when the axioms of mathematics were being established in terms of sets and functions (see Kline (1980)), the simplifying decision to use sets, rather than the more general multisets, led to a neglect of the latter. A century later, it is now recognised that multisets are merely a notational complication to the 'Zermelo/Fraenkel/Axiom-of-Choice' framework, not an illegitimacy. In describing the history, Blizard (1989) shows many situations in mathematics where multisets occur naturally.

Intensities: Under our assumption that the tessellation is stationary, the centroids of all members in a (multi)set of bounded objects form a stationary point process on the plane, but this process might also have point multiplicity and, if so, it would not be a simple point process. Nevertheless we can always define a point-process intensity.

Definition 4. The intensity of objects belonging to class $\mathrm{X}$ of bounded objects is the expected number of centroids, of the elements of $\mathrm{X}$, in any domain of unit area. It is denoted by $\lambda_{\mathrm{x}}$.

Note that, because lines are unbounded objects without centroids, we do not define $\lambda_{\mathrm{L}}$ - and likewise for any other class containing unbounded objects.
One of the intensities is needed as a scale parameter - and we have chosen $\lambda_{V}$ to play this role. We assume that

$$
0<\lambda_{\mathrm{V}}<\infty .
$$

Introducing parameters $\phi$ and $\theta$ : We now define two important parameters that quantify vertex features.

Definition 5. We denote the 'proportion of vertices that are $\pi$-vertices' by the parameter $\phi$. Equivalently, $\phi$ is the probability that the typical vertex is a $\pi$-vertex.

Another fundamental parameter of the random tessellation is $\theta$.

Definition 6. The number of edges emanating from a vertex is called the valency of that vertex. The average valency of the typical vertex is denoted by $\theta$.

In an earlier study (Weiss and Cowan, 2011), we proved the following constraints,

$$
0 \leq \phi \leq 1 \quad \text { and } \quad 3 \leq \theta \leq 6-2 \phi,
$$

and showed that the other main intensities are expressed as follows in terms of $\lambda_{V}, \theta$ and $\phi$ :

$\lambda_{\mathrm{E}}=\frac{\theta}{2} \lambda_{\mathrm{V}}, \quad \lambda_{\mathrm{Z}}=\frac{\theta-2}{2} \lambda_{\mathrm{V}}, \quad \lambda_{\mathrm{S}}=\lambda_{\mathrm{C}}=(\theta-\phi) \lambda_{\mathrm{V}}$.

We also showed that the expected number of edges contained in the typical cell-side, is $\theta /(\theta-\phi)$.

A metric parameter: There are many metric entities in a tessellation, for example, lengths of various line-segments, perimeters of cells and areas of cells. For this paper, we need to introduce just one basic metric parameter: $\bar{\ell}_{E}$, the expected length of the typical tessellation edge. The symbol $\bar{\ell}_{X}$ is introduced to be the expected length of the typical line-segment from some class $X$ of line-segments, but if we find a value of $\bar{\ell}_{X}$ for examples of $X$, it will be in terms of $\bar{\ell}_{E}$.

We assume that

$$
0<\bar{\ell}_{\mathrm{E}}<\infty .
$$

So Eqs. 1 and 4 are the two first-moment assumptions that we anticipated at the end of our Introduction.

Remark 3. Clearly, all of the intensities, $\lambda_{\mathrm{E}}, \lambda_{\mathrm{Z}}, \lambda_{\mathrm{S}}$ and $\lambda_{C}$ given in Eq. 3, are positive and finite. This follows from the finite bounds on $\theta$ and $\phi$ given by Eq. 2 together with the identities in Eq. 3 and our assumption Eq. 1 that $0<\lambda_{V}<\infty$. Similarly, our assumption Eq. 4 combined with Eq. 2 shows that all the expected lengths $\bar{\ell}_{\mathrm{X}}$ (that are proportional to $\bar{\ell}_{\mathrm{E}}$, with the proportionality constant a function of $\theta$ and/or $\phi)$ are finite and positive. For example, it is shown in Cowan and Thäle (2014) that, when $\mathrm{X}=\mathrm{S}$, $\bar{\ell}_{\mathrm{S}}=\theta \bar{\ell}_{\mathrm{E}} /(\theta-\phi)$, so clearly $\bar{\ell}_{\mathrm{S}}$ is finite and positive. 
The concept of 'adjacency': This concept, which has a unifying role in tessellation theory, is defined as follows.

Definition 7. An object $\mathrm{x} \in \mathrm{X}$ is said to be adjacent to an object $\mathrm{y} \in \mathrm{Y}$ if either $\mathrm{x} \subseteq \mathrm{y}$ or $\mathrm{y} \subseteq \mathrm{x}$. For any $\mathrm{x} \in \mathrm{X}$, the number of objects of type $\mathrm{Y}$ adjacent to $\mathrm{x}$ is denoted by $m_{\mathrm{Y}}(\mathrm{x})$. For a random tessellation we define $\mu_{\mathrm{XY}}$ as the expected value of $m_{\mathrm{Y}}(\mathrm{x})$ when $\mathrm{x}$ is the typical member of $\mathrm{X}$. In this notation, we envisage the centroid of the member in $X$ as our 'position of viewing the tessellation', and the objects in $\mathrm{Y}$ as those that we count.

Formally, we write $\mu_{\mathrm{XY}}:=\mathbb{E}_{\mathrm{X}}\left(m_{\mathrm{Y}}(\mathrm{x})\right)=$ $\int m_{\mathrm{Y}}(\mathrm{X}) \mathbb{P}_{\mathrm{X}}(d \mathrm{x})$, where $\mathbb{E}_{\mathrm{X}}$ denotes an expectation for the typical object of type $\mathrm{X}$ (that is, defined with respect to the Palm measure $\mathbb{P}_{\mathrm{X}}$; see Chiu et al. (2013)).

If $B(r)$ is the ball of radius $r$ centred at the origin, $\mu_{\mathrm{XY}}$ can also be defined formally as

$$
\lim _{r \rightarrow \infty} \frac{\sum_{\{\mathrm{x} \in \mathrm{X} \text { : centroid of } \mathrm{x} \text { is } \in B(r)\}} m_{\mathrm{Y}}(\mathrm{x})}{\text { number of } \mathrm{x} \in \mathrm{X} \text { with centroid } \in B(r)},
$$

when the limit shown is a constant; see Cowan (1978; 1980).

We can now present an alternative definition of $\phi$ using the adjacency concept and Remark 1 .

Remark 4. A vertex is a $\pi$-vertex if and only if it is adjacent to the interior of a cell-side. In this context, being 'adjacent to' is equivalent to being 'contained in'. Thus we can write $\phi$ using the adjacency notation.

$$
\phi:=\mu_{\mathrm{V}} \stackrel{\circ}{\mathrm{S}}
$$

the expected number of 'side-interiors' adjacent to a typical vertex.

Note that $\stackrel{\circ}{\mathrm{X}}$ denotes the class comprising the interiors of objects in class $X$; also $\lambda_{X}^{\circ}:=\lambda_{X}$. Thus $\stackrel{\circ}{S}$ in Eq. 6 denotes the (multi)set of all side-interiors.

Remark 5. The other fundamental parameter, $\theta$, can also be expressed using the adjacency notation. In words, $\theta$ equals the expected number of edges adjacent to the typical vertex. In symbols, $\theta \equiv \mu_{\mathrm{VE}}$.
Exchange formulae: We have a historical attachment to the symbol $\theta$, due to its extensive use in our earlier papers. Whilst we have continued its use in this paper, we prefer to use $\mu_{\mathrm{VE}}$ in the calculations that follow, because the letter-subscripts give reminders of which two object classes are involved in the adjacency count. As calculations often switch focus on the object type which is our position of viewing, the use of $\mu_{\mathrm{VE}}$ instead of $\theta$ can be helpful. For example, an important tool is the following identity, known as 'an exchange formula'. When $X$ and $Y$ are both primitive-element sets,

$$
\lambda_{X} \mu_{X Y}=\lambda_{Y} \mu_{Y X} .
$$

This identity also holds more generally (Weiss and Cowan, 2011). Notice that the identity involves two different positions of viewing, one from a typical $X$ object the other from a typical $Y$ object. As a trivial example, let $\mathrm{X}=\mathrm{E}$ and $\mathrm{Y}=\mathrm{V}$. Aided by the obvious identity $\mu_{\mathrm{EV}}=2$, Eq. 7 yields the usual proof of the first part of Eq. 3: $\lambda_{\mathrm{E}}=\lambda_{\mathrm{V}} \mu_{\mathrm{VE}} / \mu_{\mathrm{EV}}=\frac{1}{2} \theta \lambda_{\mathrm{V}}$. As a more elaborate exercise, we now prove that $\lambda_{S}$, the intensity of sides, is as shown in Eq. 3. We note that $\mu_{\mathrm{SE}}-\mu_{\mathrm{SV}}^{\circ}=1$. Therefore

$$
\begin{aligned}
\lambda_{\mathrm{S}} & =\lambda_{\mathrm{S}} \mu_{\mathrm{SE}}-\lambda_{\mathrm{S}} \mu_{\mathrm{SV}}^{\circ} \\
& =\lambda_{\mathrm{E}} \mu_{\mathrm{ES}}-\lambda_{\mathrm{V}} \mu_{\mathrm{VS}}^{\circ} \\
& =2 \lambda_{\mathrm{E}}-\phi \lambda_{\mathrm{V}} \\
& =\left(\mu_{\mathrm{VE}}-\phi\right) \lambda_{\mathrm{V}},
\end{aligned}
$$

using identities Eqs. 7 and 6 together with the obvious identity $\mu_{\mathrm{ES}}=2$ (each edge being adjacent to two sides).

Two Lemmas: The most important new intensity established in this paper is that of the M-segments. We shall prove the following lemma in later sections.

Lemma 1. The intensity of the point process formed by the centre-points of the $\mathrm{M}$-segments is

$$
\lambda_{\mathrm{M}}=\frac{1}{2}\left(\mu_{\mathrm{VE}}-2 \mu_{\mathrm{VM}} \stackrel{\circ}{-2} \mu_{\mathrm{VL}}\right) \lambda_{\mathrm{V}} .
$$

A second lemma, though deceptively obvious ${ }^{4}$, is also useful.

\footnotetext{
${ }^{4}$ A statistician, considering the sampling of a random vertex in $D$, might, for example, question why $\phi$ is a ratio of expectations in result (a) of Lemma 2, rather than the conditional expectation of a ratio,

$$
\frac{\text { number of } \pi \text {-vertices } \in D}{\text { number of vertices } \in D}
$$

given that the ratio's denominator is $>0$ ? He/she might also insist that $D$ should be a very large domain. There are some deep issues here concerning the motivational link between Palm probability measures and ergodic theory. Indeed, if one takes this statistical view and starts with this conditional expectation and a large $D$ tending to cover the whole plane, one can prove (a).
} 


\section{Lemma 2.}

(a) $\quad \phi=\frac{\lambda_{\mathrm{V}[\pi]}}{\lambda_{\mathrm{V}}} \quad$ and $\quad(b) \quad \phi=\mu_{\mathrm{VV}[\pi]}$.

The former is the ratio of the expected number of $\pi$ vertices in any domain $D$ to the expected number of vertices in $D$. The latter, which involves a class of points and one of its sub-classes, is a general way to write the probability that a typical member of the class is a member of the sub-class.

Proof of Lemma 2: (a) Using Eqs. 7 and 6, $\lambda_{\vee} \phi=$ $\lambda_{\mathrm{V}} \mu_{\mathrm{VS}}^{\circ}=\lambda_{\mathrm{S}}^{\circ} \mu_{\mathrm{SV}}^{\circ}$. But $\mu_{\mathrm{SV}}^{\circ}=\mu_{\mathrm{SV}[\pi]}^{\circ}$ and therefore $\lambda_{\mathrm{S}}^{\circ} \mu_{\mathrm{SV}}^{\circ}=\lambda_{\mathrm{S}}^{\circ} \mu_{\mathrm{SV}[\pi]}^{\circ}=\lambda_{\mathrm{V}[\pi]} \mu_{\mathrm{V}[\pi] \mathrm{S}}$. Because $\mu_{\mathrm{V}[\pi] \mathrm{S}}^{\circ}=1$, we have proved that $\lambda_{V} \phi=\lambda_{\mathrm{V}[\pi]}$. Thus part $(a)$ is proven.

(b) Once again we use Eq. 7. $\lambda_{\vee} \mu_{\mathrm{VV}[\pi]}=$ $\lambda_{\mathrm{V}[\pi]} \mu_{\mathrm{V}[\pi] \mathrm{V}}$. Note that $\mu_{\mathrm{V}[\pi] \mathrm{V}}=1$. Thus, using part $(a)$, the proof of part $(b)$ is complete.

\section{THE MAIN ADJACENCY RESULTS OF THIS PAPER}

Our central results on $\mathrm{M}$-segments, proved later, can be expressed as adjacencies. Via the following theorem we find formulae for $\mu_{\mathrm{ME}}$ and $\mu_{\mathrm{MS}}$, respectively the expected number of edges and cellsides adjacent to the typical M-segment (in other words, contained in the typical M-segment).

Theorem 1. For all our stationary planar tessellations,

$$
\begin{aligned}
& \mu_{\mathrm{ME}}=1+\frac{2 \mu_{\mathrm{VM}}^{\circ}}{\mu_{\mathrm{VE}}-2\left(\mu_{\mathrm{VM}}^{\circ}+\mu_{\mathrm{VL}}\right)}, \quad \text { and } \\
& \mu_{\mathrm{MS}}= \begin{cases}2 \mu_{\mathrm{ME}}, & \phi=0, \\
2\left(1+\frac{2 \mu_{\mathrm{VM}}-\phi \mu_{\mathrm{V}[\pi] \mathrm{M}}}{\mu_{\mathrm{VE}}-2\left(\mu_{\mathrm{VM}}^{\circ}+\mu_{\mathrm{VL}}\right)}\right), & \phi>0 .\end{cases}
\end{aligned}
$$

We note that $\mu_{\mathrm{V}[\pi] \mathrm{M}}$ is undefined when $\phi=0$ (as then there are no $\pi$-vertices), hence the two cases within Eq. 10.

Most of the adjacencies on the right-hand side of these identities have the typical vertex as the 'point of viewing'. For $\mu_{\mathrm{VL}}$ and $\mu_{\mathrm{VM}}^{\circ}$ we need to count the expected numbers of lines and $\mathrm{M}$-segment-interiors adjacent to the typical vertex and $\mu_{\mathrm{VE}}$, the expected valency of the typical vertex. The typical $\pi$-vertex, is also involved when the tessellation is not side-to-side, that is, when $\phi>0$.

\section{EXAMPLES}

Before proving Theorem 1 and Lemma 1 in the later sections, we present some examples which build experience of these tessellation entities and the formulae given above.

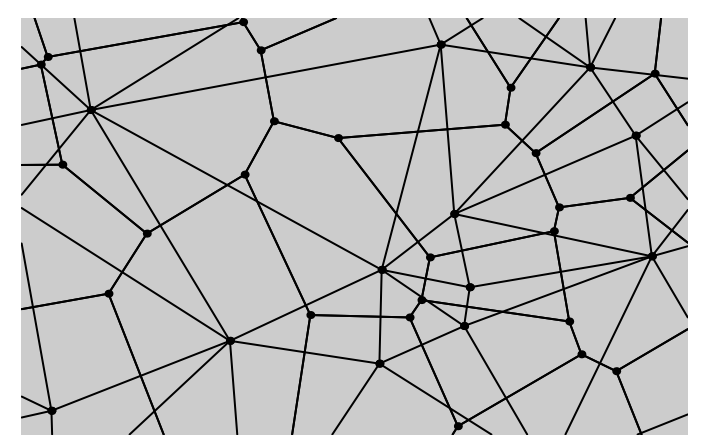

Fig. 2. The planar tessellation formed as the superposition of a Poisson-Voronoi tessellation and its Poisson-Delaunay 'dual'. See Example 1 for further definition.

Example 1. Superposition of a Poisson-Voronoi tessellation and its Delaunay dual: Superposition of two tessellations is a commonly used operation in tessellation theory. The operation is defined by saying that its frame is the union of the frames of the component tessellations. In this example illustrated by Fig. 2, the two tessellations are highly dependent, being the Voronoi and Delaunay tessellations based on the same set of seeds. The seeds are positioned according to a Poisson point process of intensity $\rho$.

The figure has been taken from Weiss and Cowan (2011), where it is shown that the vertex intensities of the Delaunay and Voronoi components are $\rho$ and $2 \rho$ respectively. Furthermore the intensity of vertices which result from frame-crossings is $4 \rho$ (a fact from Muche (2005)).

In Example 1 there are no lines, so $\mu_{\mathrm{VL}}=0$. Also there are no $\pi$-vertices, so $\phi=0, \mu_{\mathrm{V}[\pi] \mathrm{M}} \stackrel{\circ}{\text { is undefined }}$ and $\mu_{\mathrm{MS}}=2 \mu_{\mathrm{ME}}$.

The mean vertex valency in a Delaunay tessellation is 6 ; in a Voronoi, vertices have valency 3 with probability 1 . All vertices that are frame-crossings have valency 4 . So $\mu_{\mathrm{VE}}=\frac{2}{7} \cdot 3+\frac{1}{7} \cdot 6+\frac{4}{7} \cdot 4=4$. Finally, $\mu_{\mathrm{VM}} \stackrel{\circ}{\circ}=\frac{4}{7} \cdot 2=\frac{8}{7}$ because only frame-crossing vertices have adjacent $M$-segment-interiors (and 2 of them). Therefore, $\mu_{\mathrm{ME}}=1+\frac{16}{7} /\left(4-\frac{16}{7}\right)=\frac{7}{3}$. (Note that, treated individually, the Voronoi and Delaunay tessellations have $\mu_{\mathrm{VM}} \stackrel{\circ}{=} 0$ and hence $\mu_{\mathrm{ME}}=1$ and $\left.\mu_{\mathrm{MS}}=2\right)$. 


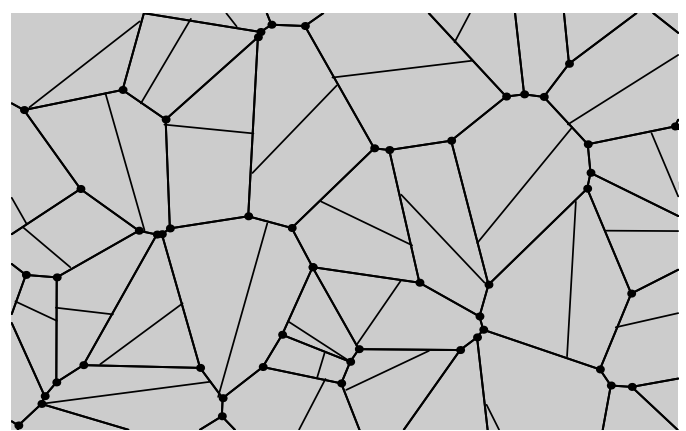

Fig. 3. A Voronoi tessellation with each cell split by a random chord through the seed used to generate that cell. These seeds are not shown; the original Voronoi vertices are shown with dots. See Example 2.

Example 2. Poisson-Voronoi cells each divided by a chord: The cells in a Poisson-Voronoi tessellation, whose seeds are a Poisson point process of intensity $\rho$, are each divided by a random chord through the cell's seed (in such a way that the chord creates two new $\pi$ vertices). See Fig. 3.

The original Voronoi vertices have intensity $2 \rho$, as do the new vertices (because each chord generates two new vertices). These new vertices are $\pi$-vertices, so $\phi=\frac{1}{2}$ and $\mu_{\mathrm{V}[\pi] \mathrm{M}} \stackrel{\circ}{ }=1$. Also each new vertex is adjacent to one $\mathrm{M}$-segment-interior while each old vertex is not adjacent to any $\mathrm{M}$-segment-interior, so $\mu_{\mathrm{VM}}^{\circ}=\frac{1}{2}$. There are no lines, so $\mathrm{L}$ is null and $\mu_{\mathrm{VL}}=0$. With probability one, all vertices have valency 3 , so $\mu_{\mathrm{VE}}=3$. Therefore $\mu_{\mathrm{ME}}=1+1 /(3-1)=\frac{3}{2}$ and $\mu_{\mathrm{MS}}=2\left(1+\left(1-\frac{1}{2} \cdot 1\right) /(3-1)\right)=\frac{5}{2}$.

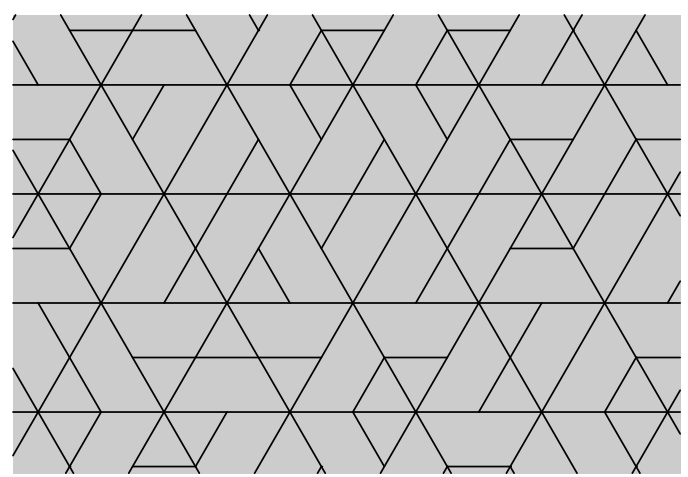

Fig. 4. A model based on the triangular lattice. See Example 3.

Example 3. A tessellation based on a triangular lattice: Consider the regular lattice of equilateral triangles as a tessellation, made random and stationary by randomly locating the origin of $\mathbb{R}^{2}$ uniformly distributed in one of the triangles. For each cell independently, draw a chord by randomly choosing two sides of the triangle and joining the midpoints of those chosen sides (see Fig. 4).
Without loss of generality, suppose that the original triangles that form the lattice have area 1 . Each original triangle has three corners; counting all of these and saying that the vertex intensity of the lattice-tessellation is ' 3 per unit area' is obviously a six-fold error (as each vertex would be counted six times). Adjusting for this, we see that the mean number of lattice tessellation vertices per unit area is $\frac{1}{2}$ and each such vertex has valency 6 . It is also adjacent to three lines and to zero M-segments.

A similar argument shows that the intensity of midpoints of the lattice triangles' sides is $\frac{3}{2}$ per unit area. Such a mid-point has

- a probability $\frac{1}{9}$ of not being a vertex of the final tessellation (because this is the chance of it not being a terminus of any random chord),

- a probability $\frac{4}{9}$ of being a $\pi$-vertex of valency 3 in the final tessellation (being the terminus of one random chord), adjacent to one line and zero $\mathrm{M}$ segments,

- a probability $\frac{4}{9}$ of being a $\bar{\pi}$-vertex of valency 4 in the final tessellation (being the terminus of two random chords), adjacent to one line and having an expected adjacency $\frac{1}{2}$ to an $\mathrm{M}$-segment-interior.

So $\frac{3}{11}$ of vertices in the final tessellation are adjacent to 3 lines and $\frac{8}{11}$ are adjacent to one line; therefore $\mu_{\mathrm{VL}}=\frac{17}{11}$. Likewise $\frac{2}{11}$ of vertices lie in the interior of one $\mathrm{M}$-segment, the remaining vertices zero. Therefore $\mu_{\mathrm{VM}} \stackrel{\circ}{=} \frac{2}{11}$. Regarding $\pi$-vertices, $\phi=\frac{4}{11}$ and $\mu_{\mathrm{V}[\pi] \mathrm{M}} \stackrel{\circ}{=}=$ 0 .

We need $\mu_{\mathrm{VE}}$ for our formulae. From the information above, $\lambda_{\mathrm{V}}=\frac{1}{2}+\frac{3}{2}\left(\frac{4}{9}+\frac{4}{9}\right)=\frac{11}{6}$ and $\mu_{\mathrm{VE}}=$ $\frac{3}{11} \cdot 6+\frac{4}{11}(3+4)=\frac{46}{11}$.

Since each of the original triangles is divided into two cells, there are two cell-centroids per unit area in the final tessellation. So $\lambda_{Z}=2$ and, from Eq. 3, $\lambda_{V}=2 /\left(\mu_{V E}-2\right) \lambda_{Z}=4 /\left(\frac{46}{11}-2\right)=\frac{11}{6}$. This provides a check on the earlier calculation of $\lambda_{v}$.

We can now find $\mu_{\mathrm{ME}}$ and $\mu_{\mathrm{MS}}$.

$$
\begin{aligned}
& \mu_{\mathrm{ME}}=1+\frac{2 \cdot \frac{2}{11}}{\frac{46}{11}-2\left(\frac{2}{11}+\frac{17}{11}\right)}=\frac{3}{2}, \\
& \mu_{\mathrm{MS}}=2 \mu_{\mathrm{ME}},
\end{aligned}
$$

because $\mu_{\mathrm{V}[\pi] \mathrm{M}}=0$. This is consistent with another simple calculation (available in this example, but not generally) which shows that the number of edges in a typical M-segment is distributed geometrically on $\{1,2, \ldots\}$ with mean $\frac{3}{2}$. 


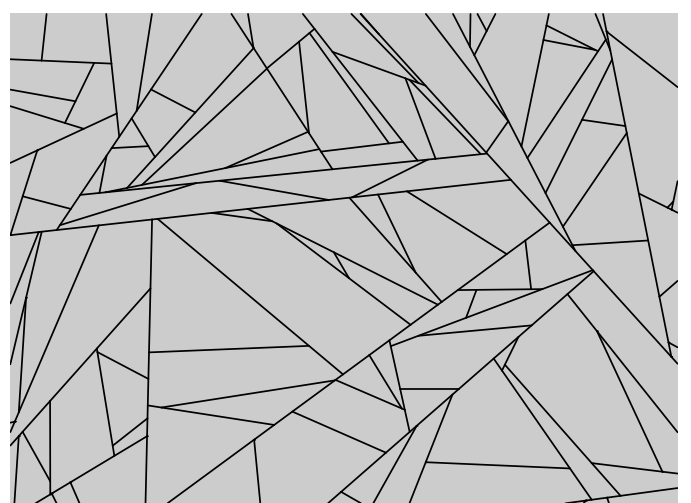

Fig. 5. Topology like the STIT tessellation. See Example 4.

Example 4. The STIT tessellation and others similar. The STIT model, which plays a central role in tessellation theory, has no lines and all vertices are $\pi$-vertices of valency 3. So all M-segments are Isegments; see Fig. 5. Some other models, for example the Gilbert model (Gilbert, 1967), the Arak-style model studied by Miles and Mackisack (2002) and many of the iteratively-divided models in Cowan (2010) (of which STIT is an important example), also have these features.

So $\phi=1$ and $\mu_{\mathrm{VE}}=3$ for this class of tessellations. Also $\mu_{\mathrm{V}[\pi] \mathrm{M}}=1$ and $\mu_{\mathrm{VL}}=0$ because the class $\mathrm{L}$ is empty. Therefore, for all these no-lines models where all vertices are $\pi$-vertices of valency $3, \mu_{\mathrm{ME}}=1+$ $2 /(3-2)=3$ and $\mu_{\mathrm{MS}}=2(1+(2-1) /(3-2))=4$.

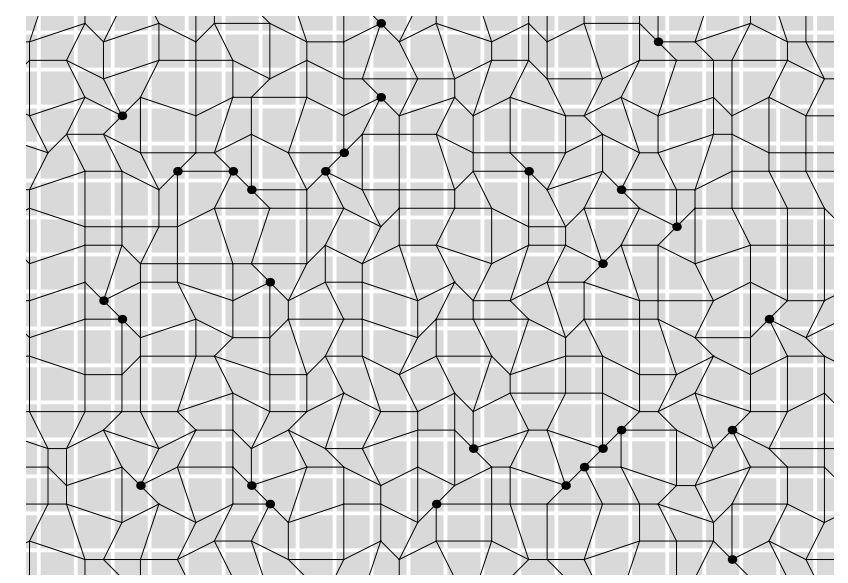

Fig. 6. The tessellation frame of Example 5 is shown in black. A black dot at a vertex indicates that this vertex is a $\pi$-vertex. The square lattice prior to the migration of vertices is shown in white.

Example 5. Migrating vertices: The construction of this example begins with the standard lattice of unitside squares, made into a stationary tessellation by the usual device of placing the origin uniformly distributed inside one of the squares. Then independently for each vertex of the tessellation a random movement is created, moving a vertex $\mathrm{v}$ originally at $(x, y)$ to $\left(x \pm \frac{1}{4}, y \pm \frac{1}{4}\right)$. The four possible new positions each have probability $\frac{1}{4}$. See Fig. 6 . Note that every vertex has valency four, so our new tessellation has $\mu_{\mathrm{VE}}=4$.

Also we have no infinite lines, so $\mathrm{L}=\emptyset$ and $\mu_{\mathrm{VL}}=0$.

We focus on the case $\mathrm{v}=(x, y)$, seen in the centre of Fig. 7, moving north-east (NE) to $\left(x+\frac{1}{4}, y+\frac{1}{4}\right)$. The figure also displays the four 'neighbouring' vertices (those connected to $v$ by an edge).

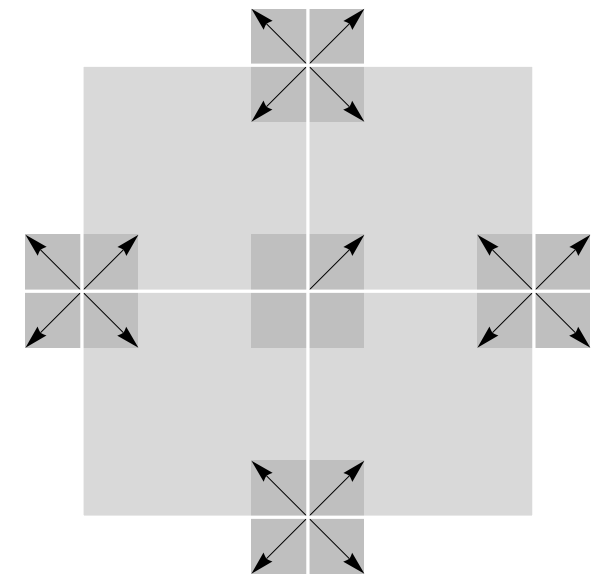

Fig. 7. As indicated by the arrows, there are four possible movements at each 'neighbour' of our vertex $\mathrm{v}$, which we assume moves $N E$ from its initial position $(x, y)$.

Fig. 7 demonstrates that there are $4^{4}$ (or 256) different changes to the positions of v's neighbours.

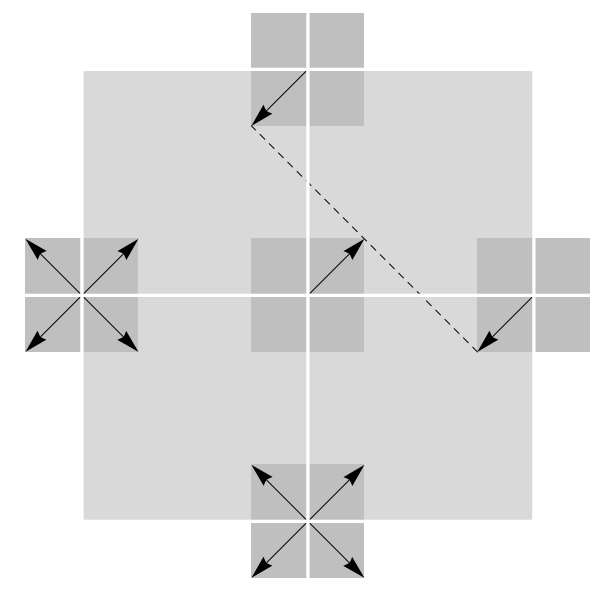

Fig. 8. The North and East neighbours have been given a SW movement $\swarrow$. This is the only way that the central vertex $\vee$ can become, post-migration, $a \pi$ vertex. The remaining two neighbours still have $4^{2}$ different changes of position. Thus the probability that the arbitrary vertex $\vee$ is a $\pi$-vertex is $4^{2} / 4^{4}$. 
Fig. 8 shows that the post-migration $\phi=\frac{16}{256}=\frac{1}{16}$. Note that a $\pi$-vertex is created in Fig. 8 at the vertex $\checkmark$ because it and the two vertices given a $\swarrow$ movement are collinear. This fact is emphasised by our dashed line-segment in Fig. 8. In this figure, we also note that $v$ lies in the interior of exactly one $\mathrm{M}$-segment interior. Thus $\mu_{\mathrm{V}[\pi] \mathrm{M}}=1$.

For the calculation of the only remaining input to Theorem 1, namely $\mu_{\mathrm{VM}}$, we present one more arrow diagram: Fig. 9. It leads to the table below - which gives the number $n_{j}$ of changes of position whereby $\mathrm{v}$ lies in the interior of $j \mathrm{M}$-segments.

\begin{tabular}{cccc}
\hline$j$ & 0 & 1 & 2 \\
\hline$n_{j}$ & 128 & 112 & 16 \\
\hline
\end{tabular}

Showing that $n_{2}=16$ : Fig. 9 shows the only cases where our vertex $v$ lies in the interior of two $\mathrm{M}$-segment interiors, of which the two dashed linesegmentss are part. Note that, to achieve this, the East and West neighbouring vertices are each permitted only two movements, $\nwarrow$ and $\nearrow$, whilst the other two (the North and South neighbours of $v$ ) are permitted only $\searrow$ and $\nearrow$. Thus there are $2^{4}$ (or 16) different changes of position allowed.

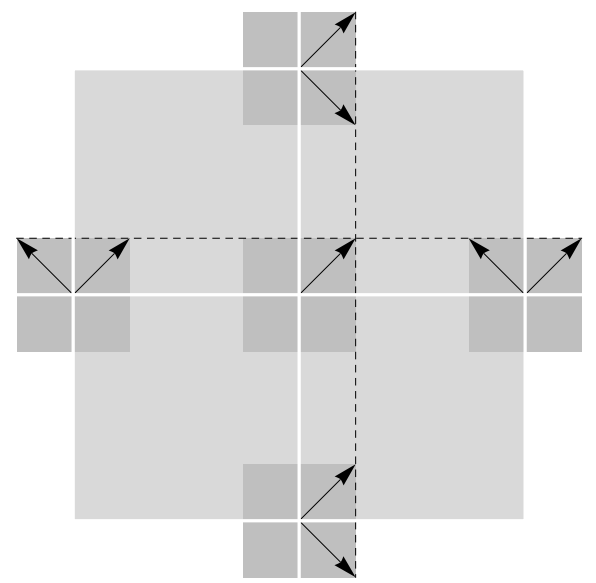

Fig. 9. The two dashed line-segments each contain the migrated position of the central vertex $\mathrm{v}$.

Showing that $n_{1}=112$ and $n_{0}=128$ : The horizontal line-segment in Fig. 9 occurs only when both the East and West neighbours of $v$ have arrows $\nwarrow$, implying $2^{2}$ possible movements for these two neighbours. But, in total, there are $4^{2}$ possible movements for them. Thus there are $4^{2}-2^{2}=12$ possible movements for the East and West neighbours where the horizontal dashed line does not appear. If the situation for the North and South neighbours remains as in Fig. 9, with $2^{2}$ ways, we can say that there are 48 ways when $v$ lies in only one $\mathrm{M}$-segment interior, it being vertical.

A similar argument shows that there are also 48 ways when $\mathrm{v}$ lies in only one M-segment interior, it being horizontal. Taking into account the 16 combinations discussed above where $\mathrm{v}$ is a $\pi$ vertex, we conclude that $n_{1}=48+48+16=112$. Furthermore, we have already shown that $n_{0}+n_{1}+$ $n_{2}=256$. Therefore $n_{0}=128$.

Thus we obtain from the $n_{j}$ table

$$
\mu_{\mathrm{VM}}=\frac{128 \cdot 0+112 \cdot 1+16 \cdot 2}{256}=\frac{9}{16} \text {. }
$$

Using Theorem 1 we can calculate $\mu_{\mathrm{ME}}$ and $\mu_{\mathrm{MS}}$ for this example as follows:

$$
\begin{aligned}
& \mu_{\mathrm{ME}}=1+\frac{2 \cdot \frac{9}{16}}{4-2 \cdot \frac{9}{16}}=\frac{32}{23} \\
& \mu_{\mathrm{MS}}=2\left(1+\frac{2 \cdot \frac{9}{16}-\frac{1}{16} \cdot 1}{4-2 \cdot \frac{9}{16}}\right)=\frac{63}{23} .
\end{aligned}
$$

In some of our examples, for instance in the one just analysed, we think that the entities $\mu_{\mathrm{ME}}$ and $\mu_{\mathrm{MS}}$ are more difficult to find than the entities $\mu_{\mathrm{VM}}, \mu_{\mathrm{VL}}$ and $\mu_{\mathrm{V}[\pi] \mathrm{M}}$ on the right-hand-side of our Theorem's identities. In the next example, we acknowledge that the reverse is true; a direct argument exists to find $\mu_{\mathrm{ME}}$ and $\mu_{\mathrm{MS}}$ without firstly evaluating the other three entities.

Example 6. Brick wall with random $H$ and $V$ chords: In this example a stationary brick-wall tessellation (using $\ell \times 1$ bricks) is modified by the independent insertion into each brick of $n$ independent random chords, sampled uniformly from the set of all horizontal or vertical chords. See Fig. 10, where $n=3$ and $\ell=2$; the chords are shown as white. The chordcreation rule implies that chords are horizontal with probability $p$ given by Eq. 11, otherwise vertical.

$$
p=\frac{1}{\ell+1} .
$$




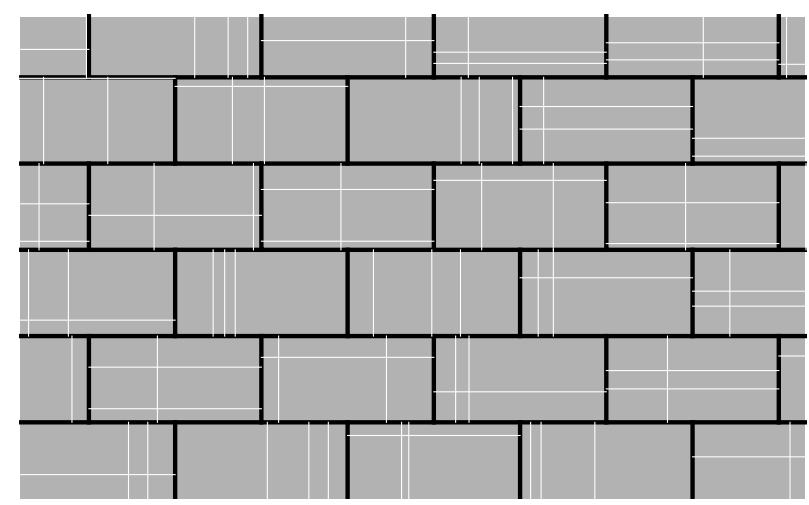

Fig. 10. The bricks in Example 6, with white chords; here $\ell=2$ and $n=3$.

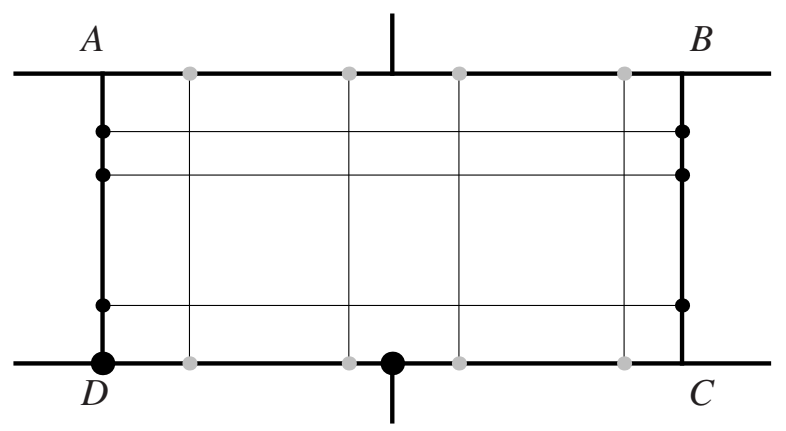

Fig. 11. One brick of Example 6 shown as the rectangle $A B C D$; here $n=7$.

Consider a typical brick $b$ from the set $B$ of bricks. One rendition of $\mathrm{b}$ when $n=7$ is the rectangle $A B C D$ in Fig. 11.

Define $H(b)$ as the number of horizontal chords of brick b; we note that $H(\mathrm{~b})$ is binomially distributed with $\mathbb{E}_{\mathrm{B}}(H(\mathrm{~b}))=n p$. Given $H(\mathrm{~b})=h$, the number of chord-crossings within b is $h(n-h)$. Unconditionally, the expected number is $\mathbb{E}_{\mathrm{B}}(h(n-h))$ which equals $n(n-1) p(1-p)$.

Prior to the chords being added, each brick has six vertices on its boundary but, in order to avoid triple counting of these, we associate only two of these with a particular brick; if $\mathrm{b}$ is $A B C D$ in the figure, these are the two vertices with large black dots. The $2 n$ vertices with smaller dots terminate the $n$ chords whilst the vertices at chord-crossings have no dot. The expected total number of vertices associated with a brick is therefore $2+2 n+n(n-1) p(1-p)$ of which $2+2 n$ will be $\pi$ vertices. So the vertex and $\pi$-vertex intensities are

$$
\begin{aligned}
\lambda_{\mathrm{V}} & =\lambda_{\mathrm{B}} \mathbb{E}_{\mathrm{B}}(2+2 n+h(n-h)) \\
& =\lambda_{\mathrm{B}}[2(n+1)+n(n-1) p(1-p)], \quad \text { and } \\
\lambda_{\mathrm{V}[\pi]} & =\mathbb{E}_{\mathrm{B}}(2+2 n)=2 \lambda_{\mathrm{B}}(n+1),
\end{aligned}
$$

where, because the brick has area $\ell, \lambda_{\mathrm{B}}=1 / \ell$. Therefore

$$
\phi=\frac{2(n+1)}{2(n+1)+n(n-1) p(1-p)} \text { and } \mu_{\mathrm{VE}}=4-\phi,
$$

the latter result following because all $\pi$-vertices have valency 3 and all non $\pi$-vertices have valency 4 in this tessellation.

To obtain $\mu_{\mathrm{VL}}$, we note that no vertex lies on $>1$ line; some (the grey dots and the two large black dots in Fig. 11) lie on 1 line, others (the small black dots) lie on no line. For brick b, given $H(\mathrm{~b})=h$, the vertical chords create $2(n-h)$ grey dots. So, given $H(\mathrm{~b})=h$, b contributes in total $2+2(n-h)$ vertices that are adjacent to one line $\in L$. Unconditionally, we expect the contribution from $\mathrm{b}$ to be $\mathbb{E}_{\mathrm{B}}(2+2 n-2 h)=2+$ $2 n-2 n p$. Thus $\lambda_{\mathrm{V}[L, 1]}$, the intensity of vertices lying on exactly 1 line, is $\lambda_{\mathrm{B}}(2+2 n-2 n p)$. Therefore

$$
\begin{aligned}
\mu_{\mathrm{VL}} & =\frac{\lambda_{\mathrm{V}[L, 1]}}{\lambda_{\mathrm{V}}}=\frac{2(1+n-n p)}{2(n+1)+n(n-1) p(1-p)} \\
& =\frac{2(1+\ell+\ell n)(\ell+1)}{2(n+1)(\ell+1)^{2}+n(n-1) \ell}, \quad \text { using Eq. } 11 .
\end{aligned}
$$

Likewise given $H(\mathrm{~b})=h$, the brick b contributes $h(n-$ $h$ ) vertices (the chord crossings) lying in the interior of two M-segments and $2 h$ vertices (those small black dots where a horizontal chord hits the boundary of $b$ ) lying on just one $\mathrm{M}$-segment interior. So, if we denote the set of vertices lying in the interior of $j \mathrm{M}$-segments by $\mathrm{V}[\stackrel{\circ}{\mathrm{M}}, j]$, then

$$
\begin{aligned}
& \lambda_{\mathrm{V}[\stackrel{\circ}{\mathrm{M}}, j]}=0, \quad \text { if } j \geq 3, \\
& \lambda_{\mathrm{V}[\stackrel{\circ}{\mathrm{M}}, 2]}=\lambda_{\mathrm{B}} \mathbb{E}_{\mathrm{B}}(h(n-h)), \quad \text { and } \\
& \lambda_{\mathrm{V}[\stackrel{\circ}{\mathrm{M}}, 1]}=\lambda_{\mathrm{B}} \mathbb{E}_{\mathrm{B}}(2 h) .
\end{aligned}
$$

Thus

$$
\begin{aligned}
\mu_{\mathrm{VM}} & =\frac{\lambda_{\mathrm{V}[\stackrel{\circ}{\mathrm{M}, 1]}}+2 \lambda_{\mathrm{V}[\stackrel{\circ}{\mathrm{M}, 2]}]}}{\lambda_{\mathrm{V}}} \\
& =\frac{\mathbb{E}_{\mathrm{B}}(2 h)+2 \mathbb{E}_{\mathrm{B}}(h(n-h))}{2(n+1)+n(n-1) p(1-p)} \\
& =\frac{2 n p+2 n(n-1) p(1-p)}{2(n+1)+n(n-1) p(1-p)} \\
& =\frac{2 n(\ell+1+\ell(n-1))}{2(n+1)(\ell+1)^{2}+n(n-1) \ell} .
\end{aligned}
$$

With similar accountancy,

$$
\begin{aligned}
& \lambda_{\mathrm{V}[\pi][\stackrel{\circ}{\mathrm{M}}, 1]}=\mathbb{E}_{\mathrm{B}}(2 h)=2 n p \lambda_{\mathrm{B}}, \quad \text { and } \\
& \lambda_{\mathrm{V}[\pi][\stackrel{\circ}{\mathrm{M}}, j]}=0 \quad \text { if } j>1 .
\end{aligned}
$$


Therefore

$$
\mu_{\mathrm{V}[\pi] \mathrm{M}}=\frac{\lambda_{\mathrm{V}[\pi][\stackrel{\circ}{\mathrm{M}}, 1]}}{\lambda_{\mathrm{V}[\pi]}}=\frac{n p}{n+1}=\frac{n}{(n+1)(\ell+1)} .
$$

Application of Eqs. 9 and 10 in Theorem 1 gives values for $\mu_{\mathrm{ME}}$ and $\mu_{\mathrm{MS}}$.

$$
\begin{aligned}
& \mu_{\mathrm{ME}}=1+\frac{2 n(1+\ell n)}{(n+1)(1+\ell)^{2}} \\
& \mu_{\mathrm{MS}}=2+\frac{2 n(1-\ell+2 \ell n)}{(n+1)(1+\ell)^{2}} .
\end{aligned}
$$

In Fig. $10, n=3$ and $\ell=2$; thus $\mu_{\mathrm{ME}}=\frac{13}{6}$ and $\mu_{\mathrm{MS}}=$ $\frac{23}{6}$.

Checking these values: To calculate $\mu_{\mathrm{ME}}$ directly, we say that each brick 'owns' $n+1 \mathrm{M}$-segments of the tessellation. These are the $n$ chords of the brick together with the left side of the brick (the brick's right side being owned by the neighbouring brick). So a typical M-segment of the tessellation can be found by considering a typical brick $b$ from the set $B$ of bricks and randomly selecting one of that brick's $n+1 \mathrm{M}$ segments.

Note that b's left side is hit from the right direction by an expected number $n p$ of chords. It is also hit by an expected number $n p$ of chords from the left direction (chords of the cell to b's left). Thus b's left side comprises an expected $1+2 n p$ edges. Conditional upon $H(\mathrm{~b})=h$, there are $h(n-h)$ crossings of b's chords and these chords contain a total of $(h+1)(n-$ $h)+h(n-h+1)=n+2 n h-2 h^{2}$ edges, on average $\left(n+2 n h-2 h^{2}\right) / n$ per chord. Unconditionally, the expected number of edges on a random choice of chord is $\mathbb{E}_{\mathrm{B}}\left(n+2 n h-2 h^{2}\right) / n$ which can be evaluated easily, because $H(\mathrm{~b}) \sim \operatorname{Binomial}[n, p]$; it is $1+2(n-1) p(1-$ p). Thus

$$
\begin{aligned}
\mu_{\mathrm{ME}} & =\frac{1}{n+1}(1+2 n p)+\frac{n}{n+1}(1+2(n-1) p(1-p)) \\
& =1+\frac{2 n p}{n+1}(p+n(1-p)) \\
& =1+\frac{2 n(1+\ell n)}{(n+1)(1+\ell)^{2}}, \quad \text { using Eq.11. }
\end{aligned}
$$

Similarly,

$$
\begin{aligned}
\mu_{\mathrm{MS}} & =\frac{1}{n+1}(2+2 n p)+\frac{n}{n+1}(2+4(n-1) p(1-p)) \\
& =2+\frac{2 n p}{n+1}(2 p+2 n(1-p)-1) \\
& =2+\frac{2 n(1-\ell+2 \ell n)}{(n+1)(1+\ell)^{2}} .
\end{aligned}
$$

Thus we get agreement with the values found by using Theorem 1.

\section{PROOF PRELIMINARIES}

The line-process component: At the outset in our proofs, we must recognise that some tessellations contain infinite unions of edges, these unions forming lines which extend infinitely in both directions. A line process and any tessellation constructed from a line process by adding edges and vertices or by operations like superposition or nesting, provide examples. Our Fig. 1 has lines which traverse the window and we ask the reader to think that some of these may extend infinitely in both directions.

In general, we must keep track of the complications that arise if the tessellation frame $\digamma$ contains lines and, to this end, the following definition is helpful.

Definition 8. The line-process component (LPC) of a planar tessellation is the union of all edges which are not contained in some M-segment or in some half-line. Equivalently, the LPC is the closure of the structure which remains after all M-segments and all half-lines of the tessellation are removed. Of course, many tessellations have no LPC so this remaining structure is null in these cases. If non-null, it is a line process comprising lines and the vertices formed by their intersections (if any). The collection of all the lines in the LPC gives us the set $\mathrm{L}$ defined and used earlier.

An LPC comprising just one set of parallel lines and therefore no line-intersections (or indeed other vertices) is permitted; such a structure is not a tessellation in our sense but it is a valid LPC (for example, in the 'brick wall' tessellation, seen as the black frame in Fig. 10).

The event the LPC has three or more of its lines passing through some point of the plane $\}$ has probability zero in many studies of stationary line processes, but there are exceptions (trivial examples using a triangular lattice or complicated examples as seen in Kallenberg (1977) and Mecke (1979) where three or more lines cross at some vertices of a stationary line process). The parameter $\mu_{\mathrm{VL}}$ quantifies the crossings of such lines, even in the exceptional cases of Kallenberg and Mecke, and also deals with the adjacencies between vertices and lines when other tessellation vertices lie on the lines of the LPC.

There can be other complications when $L$ is not null: edges might be subsets of a line in $L$ or subsets 
of an M-segment; vertices other than those from linecrossings may lie on a line in $L$, on the interior of an $\mathrm{M}$-segment or on a terminus of an M-segment; some of these might be $\pi$-vertices. Furthermore, a vertex of the LPC might have non-LPC edges emanating from it. We address these complications later in the paper, in the course of proving Theorem 1.

Line-segment processes: Our aim now is to prove that mention of half-lines in the subsection above is unnecessary, because effectively they cannot exist in stationary tessellations.

Some preliminaries concerning stationary processes of line-segments are required for this task. There are many such processes imbedded in a stationary tessellation, for example, we have a stationary process of edges - or of sides, or of Msegments. Line-segment processes can exist, of course, without the presence of a tessellation. A general theory, albeit with an added assumption of isotropy (statistical invariance under any planar rotation), was given in Cowan (1979).

One particular formula from that study (his formula (14) ) is useful here. A reference line $\mathscr{L}$ of the plane, chosen without dependence on the linesegment process, might cross some of the process's line-segments. The mean number crossed by a unit interval on $\mathscr{L}$ was found to be

$$
K \lambda \bar{\ell}
$$

where the constant $K$ equals $2 / \pi$. Also $\lambda$ is the generic intensity of segment mid-points and $\bar{\ell}$ is the expected length of the typical segment. Without isotropy, the constant $K$ is different, involving an integral of the orientation distribution for the random line-segments (see Chiu et al. (2013), formula (8.36)). This constant is finite and positive, except when all the line-segments of the process are parallel to the reference line; then it is zero. So, in all but this case, the expected number crossed by a unit interval on $\mathscr{L}$ is given by Eq. 13, for some appropriate positive constant $K$.

Remark 6. When we consider a line-segment process of edges in a stationary tessellation satisfying the regularity conditions given in our Remark 3, then both $\lambda_{\mathrm{E}}$ and $\bar{\ell}_{\mathrm{E}}$ are positive and finite; also it is impossible in a planar tessellation that all the edges can be parallel. So the expected number of tessellation edges crossed by a unit interval on $\mathscr{L}$ is given by Eq. 13, with $(\lambda, \bar{\ell})=\left(\lambda_{\mathrm{E}}, \bar{\ell}_{\mathrm{E}}\right)$. So this expected number is finite and positive.

Remark 7. $A$ half-line is defined as $\left\{v \in \mathbb{R}^{2}: v=\right.$ $\left.a_{0}+t a_{1}, a_{0}, a_{1} \in \mathbb{R}^{2}, t \in[0, \infty)\right\}$. The terminus of this half-line is the point $a_{0}$. A half-line contains an infinite number of subsets which are half-lines. A maximal half-line is a half-line that is not contained in any other half-line.

Maximal half-lines non-existent?: We should consider if unions of tessellation edges might form a maximal half-line in the tessellation's frame? The following lemma shows, however, that we can dismiss this idea. A stationary tessellation, conforming to the conditions of Remark 3 and containing maximal halflines within its frame, has probability zero. So none of the line-segments in Fig. 1, that have one end inside the window and the other on the window's boundary, would have an infinite extension from the latter end beyond the window. Also line-segments in the figure which intersect with the window's boundary at two points are not a subset of maximal half-lines.

Lemma 3. The frame of any stationary tessellation, having $0<\lambda_{\mathrm{E}}<\infty$ and $0<\bar{\ell}_{\mathrm{E}}<\infty$, contains maximal half-lines with probability zero.

Proof of Lemma 3: We assume the contrary position: Let $\mathbf{t}$ be a stationary random tessellation having $0<\lambda_{\mathrm{E}}<\infty$ and $0<\bar{\ell}_{\mathrm{E}}<\infty$ whose frame $\digamma$ contains maximal half-lines with probability $p>0$. We shall then establish a contradiction.

The termini of the maximal half-lines must, by the stationarity of $t$, form a stationary point process in $\mathbb{R}^{2}$.

Imagine that we replace each maximal half-line $H$ by a line-segment $\subset H$ of length $c_{1}$ covering $H$ 's terminus. This exercise can be repeated progressively doubling the lengths, $c_{j}=2^{j-1} c_{1}, j \geq 1$. For a given $j$, the collection of these 'replacement segments' forms a stationary line-segment process called $\mathscr{S}_{j}$.

Let $\mathscr{L}$ be a fixed reference line; we assume that $\mathscr{L}$ is chosen without dependence on $\digamma$. For example, we cannot take the line that covers an edge of $\digamma$ to be our $\mathscr{L}$. Let $I$ be a closed interval $\subset \mathscr{L}$ of unit length. We define $X_{j}$ be the number of linesegments of $\mathscr{S}_{j}$ which hit $I$. The sequence of random variables $\left\{X_{j}\right\}_{j \geq 1}$ is non-decreasing - once a linesegment crosses $I$, it remains crossing. Note that $\mathbb{E} X_{j} \sim$ $2^{j-1} c_{1}$; see Eq. 13 and the discussion near it. So we obtain $\lim _{j \rightarrow \infty} \mathbb{E} X_{j}=\infty$.

Of course, if all the (potential) half-lines created in the random tessellation are parallel to our fixed $\mathscr{L}$, the argument above doesn't apply. Then, the use instead of any other reference line not parallel to $\mathscr{L}$ will close this argument.

Now let $X$ be a random variable given by the number of intersection points on $I$ generated by the maximal half-lines of $\mathbf{t}$. With Remark 6 we have $\mathbb{E} X<\infty$. For any realisation $\mathrm{t}$ of $\mathbf{t}$ and for all $j$ we have $X_{j}(\mathrm{t}) \leq X(\mathrm{t})$. That yields $\mathbb{E} X_{j} \leq \mathbb{E} X$. With 
$\lim _{j \rightarrow \infty} \mathbb{E} X_{j}=\infty$ this contradicts the finiteness of $\mathbb{E} X$. The supposed existence of maximal half-lines leads to an infinite intensity of crossings of $\mathscr{L}$ whereas our tessellations produce only finite intensities. The contradiction implies that $p$ cannot be positive.

We can now delete the reference to (maximal) halflines in Definition 8. Maximal half-lines will not be considered further in the sequel.

\section{PROOF OF LEMMA 1 AND THEOREM 1}

Proof of Lemma 1: Located at a vertex $\vee \in \mathrm{V}$ are edge-termini, each of these being a 0 -face of an edge which emanates from the vertex. The expected number of edge-termini at the typical vertex is $\mu_{\mathrm{VE}}$ and so the intensity of edge-termini is $\mu_{V E} \lambda_{V}$. These edgetermini can be classified further. There are two for each line that $v$ lies on and, similarly, two for each $\mathrm{M}$ segment interior that $v$ lies in. Furthermore, there is one edge-terminus at $v$ for every $\mathrm{M}$-segment terminus that $v$ lies on. Therefore, recognising that 'lying on' or 'lying in' are adjacencies, we have an identity,

$$
\mu_{\mathrm{VE}}=\mu_{\mathrm{VM}}+2\left(\mu_{\mathrm{VM}} \stackrel{\circ}{+} \mu_{\mathrm{VL}}\right) \text {, }
$$

where $M_{0}$ is the class of $M$-segment termini. Noting that $\lambda_{\mathrm{M}}=\frac{1}{2} \lambda_{\mathrm{M}_{0}}=\frac{1}{2} \mu_{\mathrm{VM}_{0}} \lambda_{\mathrm{V}}$, we have the result in Lemma 1 , namely $\lambda_{\mathrm{M}}=\frac{1}{2}\left(\mu_{\mathrm{VE}}-2 \mu_{\mathrm{VM}} \stackrel{\circ}{-} \mu_{\mathrm{VL}}\right) \lambda_{\mathrm{V}}$.

Proof of Theorem 1: We commence with the exchange formula Eq. 7, setting $X=M$ and $Y=E$,

$$
\mu_{\mathrm{ME}}=\frac{\lambda_{\mathrm{E}}}{\lambda_{\mathrm{M}}} \mu_{\mathrm{EM}}
$$

and note that $\mu_{\mathrm{EM}}$, defined as the expected number of $M$-segments adjacent to the typical edge e $\in E$, has two other interpretations: firstly, it is the proportion of edges that lie on M-segments; secondly, it is the probability that the typical edge is contained in exactly one $\mathrm{M}$-segment. These interpretations follow because the edges on the LPC lines are not contained in any Msegment and the others are contained in exactly one. Indeed $\mu_{\mathrm{EL}}+\mu_{\mathrm{EM}}=1$, where $\mu_{\mathrm{EL}}=\mathbb{P}_{\mathrm{E}}\{\mathrm{e}$ is contained in exactly one line of the LPC $\}$. Thus, (14) becomes

$$
\begin{aligned}
\mu_{\mathrm{ME}} & =\frac{\frac{1}{2} \lambda_{\mathrm{V}} \mu_{\mathrm{VE}}}{\frac{1}{2}\left(\mu_{\mathrm{VE}}-2 \mu_{\mathrm{VM}}-2 \mu_{\mathrm{VL}}\right) \lambda_{\mathrm{V}}}\left(1-\mu_{\mathrm{EL}}\right) \\
& =\frac{\mu_{\mathrm{VE}}}{\mu_{\mathrm{VE}}-2 \mu_{\mathrm{VM}}-2 \mu_{\mathrm{VL}}}\left(1-\mu_{\mathrm{EL}}\right),
\end{aligned}
$$

where Lemma 1 provides $\lambda_{\mathrm{M}}$. Using again the accountancy applied in the proof of Lemma 1 and the formula $\lambda_{\mathrm{E}} \mu_{\mathrm{EL}}=\lambda_{\mathrm{V}} \mu_{\mathrm{VL}}$, we can write $\mu_{\mathrm{EL}}$ as $2 \mu_{\mathrm{VL}} / \mu_{\mathrm{VE}}$. Therefore Eq. 9, the first part of Theorem 1 , is proved.

Consider now an M-segment comprising only edges of type $E[2]$, that is, those that equal two cell sides (see Fig. 12 taken from Cowan and Thäle (2014) where these edge types are introduced).

Note that $k$ of these edges, and therefore the Msegment, contains $2 k$ sides. Obviously all $k-1$ vertices in the interior of this $\mathrm{M}$-segment are $\bar{\pi}$-type. Now add $j \pi$-vertices in the segment's interior, positioned arbitrarily (though not, of course, on top of the existing $\bar{\pi}$-vertices). As each $\pi$-vertex is added an extra edge is formed and a side is split into two sides. So this modified M-segment will comprise $k+j$ edges and $2 k+j$ sides. Note that the number of sides is twice the number of edges minus the number of added $\pi$ vertices. Thus we have

$$
\mu_{\mathrm{MS}}=2 \mu_{\mathrm{ME}}-\mu_{\mathrm{MV}[\pi]}^{\circ},
$$

the second term being the expected number of $\pi$-vertices adjacent to (that is, contained in) the typical M-segment interior. From Eq. 7, $\lambda_{\mathrm{M}}^{\circ} \mu_{\mathrm{MV}[\pi]}^{\circ}=$ $\lambda_{\mathrm{V}[\pi]} \mu_{\mathrm{V}[\pi] \mathrm{M}}$. Moreover, $\mu_{\mathrm{V}[\pi] \mathrm{M}} \stackrel{\circ}{\mathrm{M}}$ equals zero if the $\pi$ vertex lies on the LPC and equals one otherwise. Thus

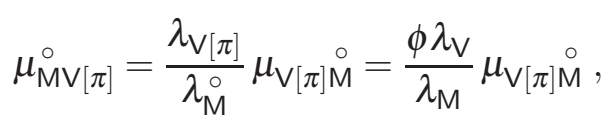

whereby Eq. 10 for $\mu_{\mathrm{MS}}$ follows, via Eq. 15 .

\section{EDGE SUBSETS}

Fig. 12, taken from Cowan and Thäle (2014), defines a classification of edges into subsets $\mathrm{E}[j], j=$ $0,1,2$ and it would be of interest to find $\mu_{\mathrm{ME}[j]}$, the expected number of edges of set $\mathrm{E}[j]$ contained in the typical M-segment.

We have found this entity (using Eq. 7 and Lemma 1) for tessellations with no LPC,

$$
\mu_{\mathrm{ME}[j]}=\frac{\lambda_{\mathrm{E}}}{\lambda_{\mathrm{M}}} \varepsilon_{j}=\frac{\mu_{\mathrm{VE}}}{\mu_{\mathrm{VE}}-2 \mu_{\mathrm{VM}}} \varepsilon_{j} .
$$

The parameters $\varepsilon_{j}$ for $j=0,1,2$, which have been introduced in Cowan and Thäle (2014) and shown to be fundamental parameters of non side-to-side tessellations, are defined to be the proportion of edges coinciding with $j$ cell sides. So $\varepsilon_{j}:=\lambda_{\mathrm{E}[j]} / \lambda_{\mathrm{E}}$ and $\varepsilon_{0}+\varepsilon_{1}+\varepsilon_{2}=1$. 


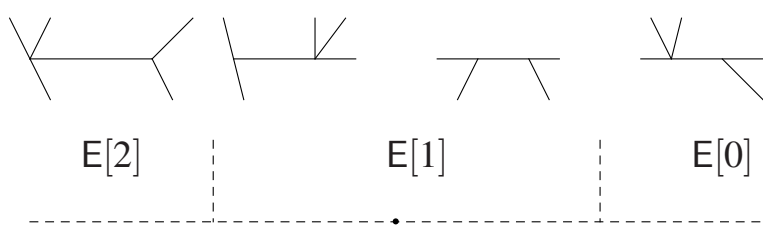

Fig. 12. In each of the four pictures, focus attention on the horizontal edge whose termini are both in view. This edge lies in the subsets shown schematically below each picture. $\mathrm{E}[j], j=0,1,2$ is the subset of edges that are equal to $j$ cell sides.

The epsilon values can be calculated for Example 5 , by focusing on a pre-migration horizontal edge e. Post-migration there are $4^{2}$ equally-likely different positions of e, but only 8 after reflection-symmetries, about a line through e's original position, are considered. In seven of these eight, e must be of type $E[2]$ regardless of how nearby vertices migrate. In the remaining one position, e now has a $\pm 45^{\circ}$ slope and it is easily seen (by considering the migrations possible for nearby vertices) that $e$ is type $E[2]$ with probability $9 / 16$, type $E[1]$ with probability $6 / 16$ and type $E[0]$ with probability $1 / 16$. So $\varepsilon_{2}=\frac{7}{8} \cdot 1+\frac{1}{8} \cdot \frac{9}{16}=\frac{121}{128}$, $\varepsilon_{1}=\frac{1}{8} \cdot \frac{6}{16}=\frac{6}{128}$, whilst $\varepsilon_{0}=\frac{1}{128}$. So,

$$
\begin{aligned}
\left\{\mu_{\mathrm{ME}[2]}, \mu_{\mathrm{ME}[1]}, \mu_{\mathrm{ME}[0]}\right\} & =\frac{4}{4-2 \cdot 9 / 16}\left\{\frac{121}{128}, \frac{6}{128}, \frac{1}{128}\right\} \\
& =\left\{\frac{121}{92}, \frac{6}{92}, \frac{1}{92}\right\},
\end{aligned}
$$

from Eq. 16.

The epsilon values are known for the STIT tessellation. See section 6.3 of Cowan (2013). So from Eq. 16, $\left\{\mu_{\mathrm{ME}[2]}, \mu_{\mathrm{ME}[1]}, \mu_{\mathrm{ME}[0]}\right\}$ equals

$$
\begin{aligned}
\frac{3}{3-2 \cdot 1} & \left\{\frac{1}{3}(8 \log 2), \frac{2}{3}(5-6 \log 2), \frac{2}{3}(2 \log 2-1)\right\} \\
& =\{8 \log 2,2(5-6 \log 2), 2(2 \log 2-1)\} \\
& \approx\{0.5452,1.6822,0.7726\} .
\end{aligned}
$$

The result in Eq. 16 could be extended to cover cases with an LPC, but only if some further parameters are introduced - and for simplicity we have elected not to do this.

\section{M-SEGMENTS IN TESSELLATIONS GENERATED BY SUPERPOSITION OR NESTING}

We will apply our results now to tessellations which can be generated by two important operations: superposition and nesting. For definitions, notations and early literature concerning these operations, we follow Cowan and Thäle (2014). Some recent applications of the operations can be seen in Neuhäuser et al (2014).

In this section the two tessellations involved are assumed to be independent and, to get reasonably pleasant formulae, a further assumption is needed: at least one of the tessellations should be isotropic.

It is difficult to find formulae for $\mu_{\mathrm{ME}}$ and $\mu_{\mathrm{MS}}$ for $\mathrm{M}$-segments in a superposition or nesting using a direct way of calculation. It is more advisable, we think, to focus on the vertices and their properties in the generated tessellation. From results for the typical vertex, other quantities such as $\mu_{\mathrm{ME}}$ and $\mu_{\mathrm{MS}}$ can be calculated - as we have done in our main theorem.

The frame $\digamma$ (the union of all edges) of a tessellation can be separated into two sets $\digamma_{\mathrm{M}}$ and $\digamma_{\mathrm{L}}$, the union of all M-segments and L-lines, respectively. The corresponding length intensities (mean frame length in any domain of unit area) are $\bar{\ell}_{\digamma}, \bar{\ell}_{F_{M}}$ and $\bar{\ell}_{\digamma_{\mathrm{L}}}$. Obviously $\digamma=\digamma_{\mathrm{M}} \cup \digamma_{\mathrm{L}}$ and $\bar{\ell}_{\digamma}=\bar{\ell}_{\digamma_{\mathrm{M}}}+\bar{\ell}_{\digamma_{\mathrm{L}}}$. For all notations related to the two tessellations we use an upper index ${ }^{(1)}$ and ${ }^{(2)}$.

Superposition: The superposition of two tessellations with frames $\digamma^{(1)}$ and $\digamma^{(2)}$ is a tessellation with frame $\digamma=\digamma^{(1)} \cup \digamma^{(2)}$, whose vertex intensity is

$$
\lambda_{V}=\lambda_{\mathrm{V}}^{(1)}+\lambda_{\mathrm{V}}^{(2)}+\frac{2}{\pi} \bar{\ell}_{\digamma}^{(1)} \bar{\ell}_{\digamma}^{(2)} .
$$

All new vertices created by superposition (these have vertex intensity $\left.2 \bar{\ell}_{\digamma}^{(1)} \bar{\ell}_{\digamma}^{(2)} / \pi\right)$ are $\times$-vertices; a superposition creates no new $\pi$-vertices. These new $\times$ vertices can be classified into three subclasses, with vertex intensities given below in brackets for each class:

- vertices adjacent to two lines and to zero Msegment interiors $\left(2 \bar{\ell}_{\digamma_{\mathrm{L}}}^{(1)} \bar{\ell}_{\digamma_{\mathrm{L}}}^{(2)} / \pi\right)$;

- vertices adjacent to one line and to one M-segment interior $\left(2\left(\bar{\ell}_{\digamma_{\mathrm{L}}}^{(1)} \bar{\ell}_{\digamma_{\mathrm{M}}}^{(2)}+\bar{\ell}_{\digamma_{\mathrm{M}}}^{(1)} \bar{\ell}_{\digamma_{\mathrm{L}}}^{(2)}\right) / \pi\right)$;

- vertices adjacent to zero lines and to two Msegment interiors $\left(2 \bar{\ell}_{\digamma_{\mathrm{M}}}^{(1)} \bar{\ell}_{\digamma_{\mathrm{M}}}^{(2)} / \pi\right)$.

All M-segments and lines of the two tessellations remain in the superposition and a superposition creates no new M-segments, that is,

$$
\digamma_{\mathrm{M}}=\digamma_{\mathrm{M}}^{(1)} \cup \digamma_{\mathrm{M}}^{(2)} \text { and } \digamma_{\mathrm{L}}=\digamma_{\mathrm{L}}^{(1)} \cup \digamma_{\mathrm{L}}^{(2)} \text {. }
$$


A superposition creates new vertices on lines and in the interior of M-segments.

Using these properties we obtain the following results

- $\quad$ for the basic parameters $\mu_{\mathrm{VE}}$ and $\phi$ :

$$
\begin{aligned}
\mu_{\mathrm{VE}} & =\frac{1}{\lambda_{\mathrm{V}}}\left(\lambda_{\mathrm{V}}^{(1)} \mu_{\mathrm{VE}}^{(1)}+\lambda_{\mathrm{V}}^{(2)} \mu_{\mathrm{VE}}^{(2)}+4 \cdot \frac{2}{\pi} \bar{\ell}_{\digamma}^{(1)} \bar{\ell}_{\digamma}^{(2)}\right) \\
\phi & =\frac{1}{\lambda_{\mathrm{V}}}\left(\lambda_{\mathrm{V}[\pi]}^{(1)}+\lambda_{\mathrm{V}[\pi]}^{(2)}\right),
\end{aligned}
$$

- for the LPC-parameter $\mu_{\mathrm{VL}}$ :

$$
\begin{aligned}
\mu_{\mathrm{VL}}= & \frac{1}{\lambda_{\mathrm{V}}}\left(\lambda_{\mathrm{V}}^{(1)} \mu_{\mathrm{VL}}^{(1)}+\lambda_{\mathrm{V}}^{(2)} \mu_{\mathrm{VL}}^{(2)}\right. \\
& +\frac{2}{\pi}\left(\bar{\ell}_{\digamma_{\mathrm{L}}}^{(1)} \bar{\ell}_{\digamma_{\mathrm{M}}}^{(2)}+\bar{\ell}_{\digamma_{\mathrm{M}}}^{(1)} \bar{\ell}_{\digamma_{\mathrm{L}}}^{(2)}\right) \\
& \left.+\frac{4}{\pi} \bar{\ell}_{\digamma_{\mathrm{L}}}^{(1)} \bar{\ell}_{\digamma_{\mathrm{L}}}^{(2)}\right),
\end{aligned}
$$

- for the M-segment parameters $\mu_{\mathrm{VM}}$ and $\mu_{\mathrm{V}[\pi] \mathrm{M}}$ :

$$
\begin{aligned}
\mu_{\mathrm{VM}}= & \frac{1}{\lambda_{\mathrm{V}}}\left(\lambda_{\mathrm{V}}^{(1)} \mu_{\mathrm{VM}}^{(1)}+\lambda_{\mathrm{V}}^{(2)} \mu_{\mathrm{VM}}^{(2)}\right. \\
& +\frac{2}{\pi}\left(\bar{\ell}_{\digamma_{\mathrm{L}}}^{(1)} \bar{\ell}_{\digamma_{\mathrm{M}}}^{(2)}+\bar{\ell}_{\digamma_{\mathrm{M}}}^{(1)} \bar{\ell}_{\digamma_{\mathrm{L}}}^{(2)}\right) \\
& \left.+\frac{4}{\pi} \bar{\ell}_{\digamma_{\mathrm{M}}}^{(1)} \bar{\ell}_{\digamma_{\mathrm{M}}}^{(2)}\right) .
\end{aligned}
$$

Nesting: To describe nesting we again start with a tessellation having frame $\digamma^{(1)}$. For each cell $z \in Z^{(1)}$ we independently generate a tessellation with frame $\digamma^{(2)}(\mathbf{z})$ distributed as $\digamma^{(2)}$ and add $\digamma^{(2)}(z) \cap z$ to $\digamma^{(1)}$. That means independent copies of $\digamma^{(2)}$ are nested into the cells of $\digamma^{(1)}$. The nested tessellation has the frame $\digamma=\digamma^{(1)} \cup \underset{z \in Z^{(1)}}{\bigcup}\left(\digamma^{(2)}(z) \cap z\right)$ and the vertex intensity

$$
\lambda_{\mathrm{V}}=\lambda_{\mathrm{V}}^{(1)}+\lambda_{\mathrm{V}}^{(2)}+\frac{4}{\pi} \bar{\ell}_{\digamma}^{(1)} \bar{\ell}_{\digamma}^{(2)} .
$$

All new vertices in a nesting (they have intensity $\left.4 \bar{\ell}_{\digamma}^{(1)} \bar{\ell}_{\digamma}^{(2)} / \pi\right)$ are $\pi$-vertices with three emanating edges. There are two subclasses:

- vertices which lie in the interior of one M-segment of $\digamma^{(1)}$ (their intensity is $4 \bar{\ell}_{\digamma_{M}}^{(1)} \bar{\ell}_{\digamma}^{(2)} / \pi$ ),

- vertices which lie on one line of $\digamma^{(1)}$ (their intensity is $\left.4 \bar{\ell}_{\digamma_{L}}^{(1)} \bar{\ell}_{\digamma}^{(2)} / \pi\right)$.

The lines and $\mathrm{M}$-segments of $\digamma^{(1)}$ remain, but the nested structure can create new vertices on them. In the interior of the cells of $\digamma^{(1)}$ new M-segments occur. The LPC of $\digamma^{(2)}$ disappears, the nested tessellation contains only lines from $\digamma^{(1)}$. Using that we obtain
- for the basic parameters $\mu_{\mathrm{VE}}$ and $\phi$ :

$$
\begin{aligned}
\mu_{\mathrm{VE}} & =\frac{1}{\lambda_{\mathrm{V}}}\left(\lambda_{\mathrm{V}}^{(1)} \mu_{\mathrm{VE}}^{(1)}+\lambda_{\mathrm{V}}^{(2)} \mu_{\mathrm{VE}}^{(2)}+3 \cdot \frac{4}{\pi} \bar{\ell}_{\digamma}^{(1)} \bar{\ell}_{\digamma}^{(2)}\right) \\
\phi & =\frac{1}{\lambda_{\mathrm{V}}}\left(\lambda_{\mathrm{V}[\pi]}^{(1)}+\lambda_{\mathrm{V}[\pi]}^{(2)}+\frac{4}{\pi} \bar{\ell}_{\digamma}^{(1)} \bar{\ell}_{\digamma}^{(2)}\right)
\end{aligned}
$$

- $\quad$ for the LPC-parameter $\mu_{\mathrm{VL}}$ :

$$
\mu_{\mathrm{VL}}=\frac{1}{\lambda_{\mathrm{V}}}\left(\lambda_{\mathrm{V}}^{(1)} \mu_{\mathrm{VL}}^{(1)}+\frac{4}{\pi} \bar{\ell}_{\digamma_{\mathrm{L}}}^{(1)} \bar{\ell}_{\digamma}^{(2)}\right)
$$

- for the M-segment parameters $\mu_{\mathrm{VM}}$ and $\mu_{\mathrm{V}[\pi] \stackrel{\circ}{\mathrm{M}}}$ :

$$
\begin{aligned}
\mu_{\mathrm{VM}}= & \frac{1}{\lambda_{\mathrm{V}}}\left(\lambda_{\mathrm{V}}^{(1)} \mu_{\mathrm{VM}}^{(1)}+\lambda_{\mathrm{V}}^{(2)}\left(\mu_{\mathrm{VM}}^{(2)}+\mu_{\mathrm{VL}}^{(2)}\right)\right. \\
& \left.+\frac{4}{\pi} \bar{\ell}_{\digamma_{\mathrm{M}}}^{(1)} \bar{\ell}_{\digamma}^{(2)}\right), \\
\mu_{\mathrm{V}[\pi] \stackrel{\mathrm{M}}{ }=} & \frac{1}{\lambda_{\mathrm{V}[\pi]}}\left(\lambda_{\mathrm{V}[\pi]}^{(1)} \mu_{\mathrm{V}[\pi] \stackrel{\circ}{(1)}}\right. \\
& \left.+\lambda_{\mathrm{V}[\pi]}^{(2)}\left(\mu_{\mathrm{V}[\pi] \stackrel{\circ}{(2)}}^{(2)}+\mu_{\mathrm{V}[\pi] \mathrm{L}}^{(2)}\right)+\frac{4}{\pi} \bar{\ell}_{\digamma}^{(1)} \bar{\ell}_{\digamma}^{(2)}\right),
\end{aligned}
$$

Using these results with Lemma 1 and Theorem 1 the characteristics of $\mathrm{M}$-segments in tessellations generated by superposition or nesting can be calculated.

Examples of these operations: In order to show some practical value, superposition and nesting are now considered for an isotropic STIT tessellation and an isotropic Poisson line tessellation (PLT). As a scaling parameter we use that the mean area of the typical cell in both tessellations is equal to one or

$$
\lambda_{\mathrm{Z}}^{(s t i t)}=\lambda_{\mathrm{Z}}^{(p l t)}=1
$$

Our compilation of the basic parameters of a STIT tessellation (which we have discussed in Example 4; see Fig. 5) are as follows. Details are omitted.

- A STIT tessellation has no LPC: $\digamma^{(s t i t)}=\digamma_{\mathrm{M}}^{(\text {stit })}$.

- All vertices are $\pi$-vertices with three emanating edges: $\phi^{(\text {stit })}=1$ and $\mu_{\mathrm{VE}}^{(\text {stit })}=3$.

- Therefore $\mu_{\mathrm{VM}}^{(\text {stit })}=\mu_{\mathrm{V}[\pi] \mathrm{M}}^{(s t i t)}=1$ and $\mu_{\mathrm{VL}}^{(\text {stit })}=0$.

- With the scaling parameter we obtain further: $\bar{\ell}_{\digamma}^{(s t i t)}=\bar{\ell}_{\digamma_{\mathrm{M}}}^{(\text {stit })}=\sqrt{\pi}, \quad \bar{\ell}_{\digamma_{\mathrm{L}}}^{(\text {stit })}=0$ and $\lambda_{\mathrm{V}}^{(\text {stit })}=2$.

The basic parameters of a Poisson line tessellation are as follows.

- A PLT has no M-segments: $\digamma^{(p l t)}=\digamma_{\mathrm{L}}^{(p l t)}$. 
- All vertices are $\times$-vertices, there are no $\pi$-vertices: $\phi^{(p l t)}=0$ and $\mu_{\mathrm{VE}}^{(p l t)}=4$.

- Therefore $\mu_{\mathrm{VM}}^{(p l t)}=0$ and $\mu_{\mathrm{VL}}^{(p l t)}=2$.

- With the scaling parameter we obtain further: $\bar{\ell}_{\digamma}^{(p l t)}=\bar{\ell}_{\digamma_{\mathrm{L}}}^{(p l t)}=\sqrt{\pi}, \quad \bar{\ell}_{\digamma_{\mathrm{M}}}^{(p l t)}=0$ and $\lambda_{\vee}^{(p l t)}=1$.

The following three examples for a STIT and a Poisson line tessellation are considered.

Example 7. A superposition of a STIT and a PLT, see Fig. 13, has the following parameters.

$$
\begin{aligned}
\lambda_{\mathrm{V}} & =5, & \phi & =\frac{2}{5}, \\
\mu_{\mathrm{VL}} & =\frac{4}{5}, & \mu_{\mathrm{VE}} & =\frac{18}{5} \\
& =\frac{4}{5}, & \mu_{\mathrm{V}[\pi] \mathrm{M}} & =1 .
\end{aligned}
$$

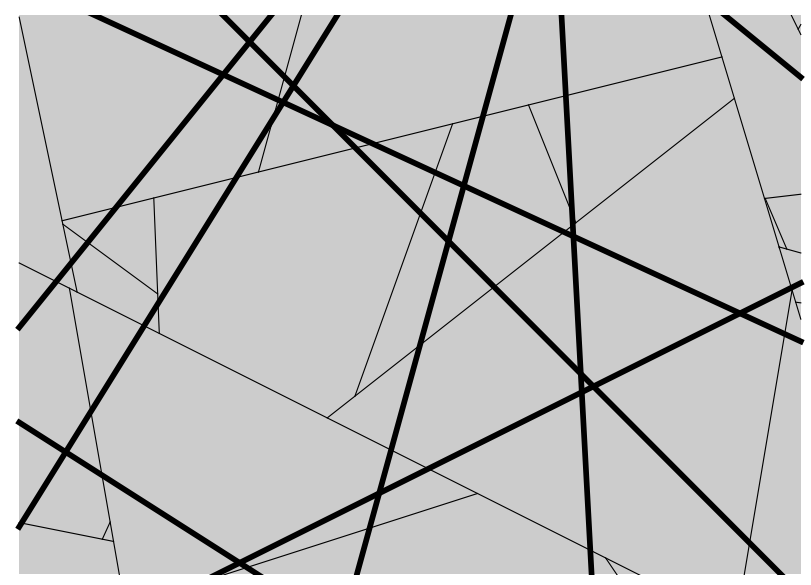

Fig. 13. The superposition of a STIT tessellation (with thin edges) and a Poisson line tessellation (thick lines).

With Lemma 1 and Theorem 1 the M-segment characteristics are

$$
\lambda_{\mathrm{M}}=1, \quad \mu_{\mathrm{ME}}=5, \quad \mu_{\mathrm{MS}}=8 .
$$

Example 8. A nesting of a STIT into the cells of a PLT, see Fig. 14, creates a tessellation with the following parameters

$$
\begin{aligned}
\lambda_{\mathrm{V}} & =7, & \phi & =\frac{6}{7}, \\
\mu_{\mathrm{VL}} & =\frac{6}{7}, & \mu_{\mathrm{VE}} & =\frac{22}{7} \\
& =\frac{2}{7}, & \mu_{\mathrm{V}[\pi] \mathrm{M}} & =\frac{2}{6}
\end{aligned}
$$

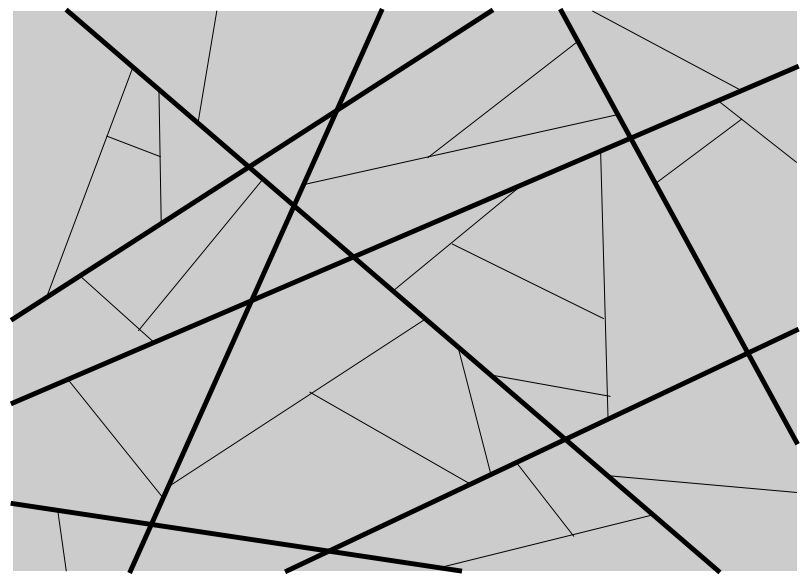

Fig. 14. A STIT tessellation (with thin edges) nested into a Poisson line tessellation (thick lines).

With Lemma 1 and Theorem 1 the M-segment characteristics are

$$
\lambda_{\mathrm{M}}=3, \quad \mu_{\mathrm{ME}}=\frac{5}{3}, \quad \mu_{\mathrm{MS}}=\frac{8}{3} .
$$

Example 9. A nesting of a PLT into the cells of a STIT tessellation, see Fig. 15, creates a nested tessellation with no LPC having the following parameters.

$$
\begin{aligned}
& \lambda_{\mathrm{V}}=7, \quad \phi=\frac{6}{7}, \quad \mu_{\mathrm{VE}}=\frac{22}{7}, \\
& \mu_{\mathrm{VL}}=0, \quad \mu_{\mathrm{VM}}=\frac{8}{7}, \quad \mu_{\mathrm{V}[\pi] \stackrel{\mathrm{M}}{\circ}}=1 .
\end{aligned}
$$

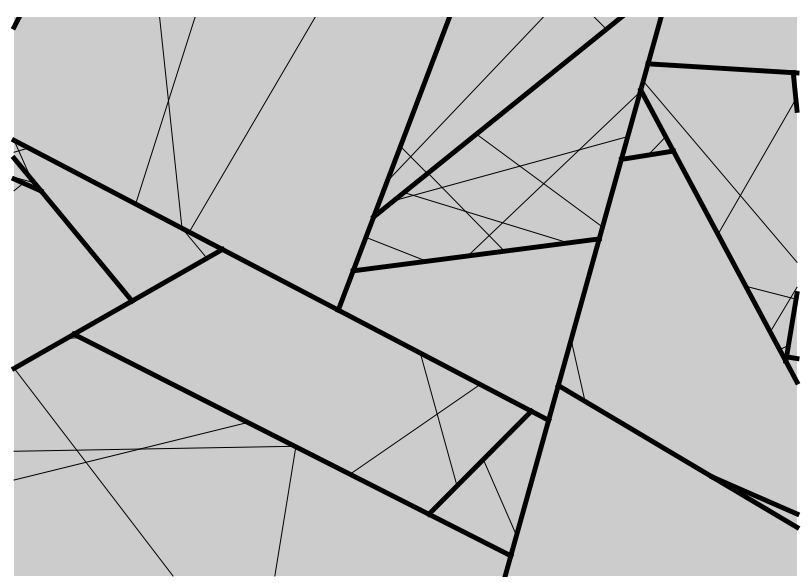

Fig. 15. A Poisson line tessellation (with thin edges) nested into a STIT tessellation (thick lines).

With Lemma 1 and Theorem 1 the M-segment characteristics are

$$
\lambda_{\mathrm{M}}=3, \quad \mu_{\mathrm{ME}}=\frac{11}{3}, \quad \mu_{\mathrm{MS}}=\frac{16}{3} .
$$




\section{SOME CONCLUDING COMMENT}

1. For a tessellation where all M-segments are Isegments and where all $\pi$-vertices have three emanating edges we have

$$
2 \lambda_{\mathrm{M}}=\lambda_{\mathrm{V}[\pi]}=\phi \lambda_{\mathrm{V}}
$$

because the termini of an $\mathrm{M}$-segment are $\pi$ vertices and any $\pi$-vertex is a terminus of one $\mathrm{M}$ segment.

2. If the tessellation is side-to-side (that is, if $\phi=0$ ) or if $\phi>0$ and all the $\pi$-vertices are lying on lines (that is $\mu_{\mathrm{V}[\pi] \mathrm{M}}=0$ ), then we have (using Theorem 1) that

$$
\mu_{\mathrm{MS}}=2 \mu_{\mathrm{ME}} .
$$

3. Vertices in the interior of the typical M-segment: $\mu_{\mathrm{MV}}=\mu_{\mathrm{ME}}-1$ and, with the exchange formula Eq. 7 and Lemma 1,

$$
\mu_{\mathrm{MV}[\pi]}=\frac{\phi \lambda_{\mathrm{V}}}{\lambda_{\mathrm{M}}} \mu_{\mathrm{V}[\pi] \stackrel{\mathrm{M}}{ }}=2 \frac{\phi \mu_{\mathrm{V}[\pi] \stackrel{\circ}{\mathrm{M}}}}{\mu_{\mathrm{VE}}-2\left(\mu_{\mathrm{VM}}+\mu_{\mathrm{VL}}\right)} .
$$

4. The proportions of edges which lie on M-segments or on lines: Using Eq. 9 and Lemma 1, it follows that

$$
\mu_{\mathrm{EM}}=\frac{\lambda_{\mathrm{M}}}{\lambda_{\mathrm{E}} \mu_{\mathrm{ME}}}=\frac{\mu_{\mathrm{VE}}-2 \mu_{\mathrm{VL}}}{\mu_{\mathrm{VE}}} .
$$

From the identity $\mu_{\mathrm{EM}}+\mu_{\mathrm{EL}}=1$, we have $\mu_{\mathrm{EL}}=$ $2 \mu_{\mathrm{VL}} / \mu_{\mathrm{VE}}$.

5. The proportions of cell-sides which lie on Msegments or on lines: Using Eqs. 3 and 10 for $\phi>0$,

$$
\mu_{\mathrm{SM}}=\frac{\lambda_{\mathrm{M}}}{\lambda_{\mathrm{S}}} \mu_{\mathrm{MS}}=\frac{\mu_{\mathrm{VE}}-2 \mu_{\mathrm{VL}}-\phi \mu_{\mathrm{V}[\pi] \mathrm{M}}}{\mu_{\mathrm{VE}}-\phi} .
$$

From $\mu_{\mathrm{SM}}+\mu_{\mathrm{SL}}=1$ and $\mu_{\mathrm{V}[\pi] \mathrm{M}}+\mu_{\mathrm{V}[\pi] \mathrm{L}}=1$, we can write $\mu_{\mathrm{SL}}=\left(2 \mu_{\mathrm{VL}}-\phi \mu_{\mathrm{V}[\pi] \mathrm{L}}\right) /\left(\mu_{\mathrm{VE}}-\phi\right)$.

\section{ACKNOWLEDGEMENT}

We thank both referees for their careful reading of our paper and for their excellent suggestions.

\section{REFERENCES}

Blizard WD (1989). Multiset Theory. Notre Dame J Form L 30:36-66.

Chiu SN, Stoyan D, Kendall WS, Mecke J (2013). Stochastic Geometry and its Applications. 3rd Ed. Chichester: Wiley.
Cowan R (1978). The use of ergodic theorems in random geometry. Suppl Adv Appl Probab 10:47-57.

Cowan R (1979). Homogeneous line-segment processes. Math Proc Cambridge 86:481-9.

Cowan R (1980). Properties of ergodic random mosaic processes. Math Nachr 97:89-102.

Cowan R (2010). New classes of random tessellations arising from iterative division of cells. Adv Appl Probab 42:26-47.

Cowan R (2013). Line-segments in the isotropic planar STIT tessellation. Adv Appl Probab 45:295-311.

Cowan R, Thäle C (2014). The character of planar tessellations which are not side-to-side. Image Anal Stereol 33:39-54.

Cowan R, Weiss V (2015). Constraints on the fundamental topological parameters of spatial tessellations. Math Nachr 288:540-65.

Gilbert EN (1967). Surface films of needle-shaped crystals. In: Noble B, Ed. Applications of undergraduate mathematics in engineering, 329-46. New York: Macmillan.

Kallenberg O (1977). A counterexample to R. Davidson's conjecture on line processes. Math Proc Cambridge 82:301-7.

Kline M (1980). Mathematics: The loss of certainty. Oxford: Oxford University Press.

Mackisack M, Miles RE (1996). Homogeneous rectangular tessellations. Adv Appl Probab 28:993-1013.

Mecke J (1979). An explicit description of Kallenberg's lattice type point process. Math Nachr 89:185-95.

Mecke J, Nagel W, Weiss V (2007). Length distributions of edges in planar stationary and isotropic STIT tessellations. J Contemp Math Anal 42:28-43.

Mecke J, Nagel W, Weiss V (2011). Some distributions for I-segments of planar random homogeneous STIT tessellations. Math Nachr 284:1483-95.

Miles RE, Mackisack M (2002). A large class of random tessellations with the classic Poisson polygon distributions. Forma 17:1-17.

Muche L (2005). The Poisson-Voronoi tessellation. Adv Appl Probab 37:279-96.

Neuhäuser D, Hirsch C, Gloaguen C, Schmidt V (2014). Ratio limits and simulation algorithms for the Palm version of stationary iterated tessellations. J Stat Comput Sim 84:1486-504.

Schneider R, Weil W (2008). Stochastic and integral geometry. Berlin: Springer.

Weiss V, Cowan R (2011). Topological relationships in spatial tessellations. Adv Appl Probab 43:963-84. 